\title{
Article \\ 6G Enabled Industrial Internet of Everything: Towards a Theoretical Framework
}

\author{
Prafulla Kumar Padhi 1,*(D) and Feranando Charrua-Santos ${ }^{1,2}$ (D) \\ 1 Eletromechanical Department, University of Beira Interior, 6201-001 Covilha, Portugal; bigares@ubi.pt \\ 2 C-MAST-Centre for Mechanical and Aerospace Science and Technologies, University of Beira Interior, \\ 6201-001 Covilha, Portugal \\ * Correspondence: prafulla.k.padhi@ubi.pt
}

check for updates

Citation: Padhi, P.K.;

Charrua-Santos, F. 6G Enabled

Industrial Internet of Everything:

Towards a Theoretical Framework.

Appl. Syst. Innov. 2021, 4, 11.

https://doi.org/10.3390/asi4010011

Received: 25 December 2020

Accepted: 5 February 2021

Published: 11 February 2021

Publisher's Note: MDPI stays neutral with regard to jurisdictional claims in published maps and institutional affiliations.

Copyright: (c) 2021 by the authors. Licensee MDPI, Basel, Switzerland. This article is an open access article distributed under the terms and conditions of the Creative Commons Attribution (CC BY) license (https:// creativecommons.org/licenses/by/ $4.0 /)$.

\begin{abstract}
Currently, the deficiencies of the 5G mobile system as an enabler of Internet of Everything (IoE) applications are stimulating global research activities to focus on the sixth generation (6G) wireless system. The potential of IoE is enormous and growing exponentially. With the dawn of the fifth industrial revolution, IoE is transposing into industrial Internet of Everything (IIoE) projects which are complex and are eventuating to become a prominent technology for all industries offering new opportunities. This study embodies a synthesis of 6G, IoT, IoE, IIoE exhaustive literature review advancing knowledge to facilitate theory development. For the first time, a novel theoretical framework for the 6G-enabled IIoE (henceforth referred to as 6GIIoE) system was developed. Judiciously, sequential methodology is best suited for this emerging discipline research to create significant new knowledge in the literature contributing eternal insights to expound valuable contexts to ruminate significant findings. The theoretical framework developed recognizes 6GIIoE priority areas, challenges, and applications bestowing a guide for 6G-enabled IIoE initiatives divulging opportunities for future research activities.
\end{abstract}

Keywords: 6GIIoE priorities; 6GIIoE challenges; 6GIIoE applications; information system; sequential methodology; 6GIIoE theoretical framework

\section{Introduction}

The connection of embedded devices, sensors and machines via the Internet has revolutionized the way people live, work and play. In 1999, Kevin Ashton of Massachusetts Institute of Technology (MIT-Auto ID Center) used the term "Internet of Things-IoT" [1]. The IoT connects all the devices, sensors, or machines with each other in a physical system with the Internet or Intranet or wireless communication such as 6G [2] to communicate with each other for user friendly services. The IoT era is being reinstated by the IoE [3] era, where ubiquitous connectivity is possible. IoE connectivity is a broad topic that provides different solutions for massive data to be transferred to the edge of the network. With the emergence of the IoE era, industrial control systems (ICSs) [4] (p. 1) are shifting into IIoE [5] and includes all use cases, challenges, and applications for all industries. The evolution of mobile communications has been driven primarily by continual incessant demand for mobile communication capacity for higher data rates. During the last decade, a cardinal paradigm transformation happening gradually from the rate-centric enhanced mobile broadband (eMBB) services towards ultra-low latency communications (URLLC) [6] (p. 57065) and high reliability related to IIoE system that connects billions of devices, censors, machines, and people.

The fifth generation (5G) wireless communication standardization is over, and deployment has started in many countries globally. The development of 5G [7] mobile systems reveals the immanent limitations relative to its original engagement as an enabler for internet of everything (IoE) applications. A smattering of technologies will be developed 
to play a pivotal role towards the end of the $6 \mathrm{G}$ research process to attain comprehensive specification towards a $6 \mathrm{G}$ global standard. One such eye-catching technology is quantum computing and communications (QCC) [6] (p. 57067) that can provide long distance networking and security. At present, major research pursuits are focused on the quantum technologies realm which will intersect with 6G deployment. Starting in the 2030s, to achieve sustainable competitive edge of wireless communications applications, some industries and academia have commenced to conceptualize the sixth generation (6G) foundation for the emerging communication needs. Even though there is $6 \mathrm{G}$ enthusiasm, the basic architectural and performance components of the $6 \mathrm{G}$ mobile system remain largely undefined. Hence, one of the objectives of this study is to outline a novel holistic, efficacious, cogent vision that construes the conception of a $6 \mathrm{G}$ enabling IIoE system.

$6 \mathrm{G}$, in our opinion, is not a sheer expedition of additional high-frequency bands wireless spectrum, but it will be an authentic concurrence of breathtaking latent services and emerging technological trends. The role of $6 \mathrm{G}$ communication will revolutionize IIoE with mobile Teraband reliable low latency communication (MTRLLC) [6] (p. 57069) and massive network capacity accelerating the rate of data download, enabling the of use real-time data in the industrial operations so that data can be easily shared remotely. A massive volume of data can be shared between the machines via edge (cloud) platform and can be downloaded as well as uploaded at the edge of the $6 \mathrm{G}$ network to support connected intelligent industrial applications. The information communication technology (ICT), operational technology (OT) and emerging 6G communications are going to converge to form the 6G IIoE system. The 6GIIoE system is an emerging high-performance growth engine that has inspired global research and development $(\mathrm{R} \& \mathrm{D})$ enthusiasm and opened application opportunities for the "connected intelligent industrial world" (CIIW) [8] (p. 3), [9], [10] (p. 102), [11] for the emerging fifth industrial revolution.

The literature review bestowed a synthesis of 6G, IoT/IoE priority areas, challenges, applications, architectures, and their potential influence in industries. Drawing from the exhaustive review, we developed a novel theoretical framework for the adoption and execution of $6 \mathrm{GIIoE}$ system.

\subsection{Organization and Roadmap}

The rest of the paper is organized as follows: In Section 2, we present an exhaustive literature review and synthesis. In Section 3, the theoretical foundation outlines the 6GIIoE boundaries, relevant theories that support the theoretical framework. The sequential methodology found to be the best-suited for this study is described in the Section 4 . The 6GIIoE paradigm is presented outlining $6 \mathrm{G}$ technologies enabler, new service classes and IIoE overview in Section 5. A 5-layer 6GIIoE modular architecture is proposed in Section 6. A list of 6GIIoE priority areas, applications and challenges is described in Section 7. In the Section 8, a novel theoretical framework and contributions of the framework components are discussed. Consolidated lessons learned are explained in Section 9. We outline the future research agenda and research gaps in Section 10. Recommendations are offered for the academia and practitioners in the Section 11. Section 12, finally, concludes that the developed theoretical framework falls under a theory's phenomenon that has not been covered by prior theories.

\subsection{Background and Problem Formulation}

There has been a scattering of theories proposed and developed in the IoT domain. Our exhaustive literature review demonstrates that theoretical framework on the 6GIIoE system approbation and execution is non-existent in the literature.

Researchers place an eminent value on theoretical frameworks. There is prevailing concern that the information systems (IS) [12] discipline on developing theories does not exist relevant to the discipline that deals with 6GIIoE system, operating system (OS) software solutions and topics, namely philosophy, anthropology, socioeconomic, psychology and ethical dimensions. The IS community of researchers and practitioners does not even share 
a common definition of the term 'information system (IS) and does not have an appropriate influential theory to comprehend information handling activities in human structures. To date, the IS discipline is not well understood by academia in other fields, and even in fields related to IS. Furthermore, the development of new theories and the depuration of prevailing theories have been relatively ignored within the IS field [12] (pp. 2-4, 20). Confirming the lack of research publications on theory advancement in IS discipline related to $6 \mathrm{G}$ and IIoE, an extensive review of literature produced no research papers that deals with $6 \mathrm{GIIoE}$ theoretical framework in a multi-dimensional value co-creation approach. The importance of the $6 \mathrm{GIIoE}$ system topic is gaining traction in the industrial applications bestowing enormous value on co-creation. A pressing issue in this regard is how industrial organizations can adapt their traditional structures and execute the innovation in the 6GIIoE field to create value.

To fill the above-mentioned void, this study bestows a comprehensive understanding to offer holistic guidance to develop a theoretical framework.

\subsection{Key Contributions}

- A comprehensive contribution of our research work is the creation of a novel theoretical framework on the 6GIIoE system approbation and execution which is non-existent in the literature.

- Sequential methodology is employed, for the first time, to exploit adaptive approaches of various applications that identifies the future directions in this field of $6 \mathrm{GIIoE}$ system research and development.

\section{Literature Review and Synthesis}

An exhaustive review of relevant literature is paramount in any academic research endeavor. A persuasive review generates a sound foundation for propelling new knowledge. It provides theory development and identifies where the research gap exists. Despite a great deal having been written regarding IoT, there is a captious deficit of papers on IoE research-based studies and IoE literature reviews. A detailed review of IS innovation, theoretical framework development [10] (pp. 97-108), [13] (pp. 10-12), [14] related to IoT/IoE was done. A void in the literature exist particularly regarding the 6G-enabled IIoE studies. In the Table 1 (summary of the literature review), we demonstrate the research paper classification of the relevant research papers reviewed that confirms our view that $6 \mathrm{G}$-enabled IIoE research studies are non-existent. The literature review bestows an analysis of 6G, IoT/IoE and the relevant priority areas to focus, applications, challenges, and architectures and their influence in industries. Drawing from the exhaustive literature review, we developed a novel theoretical framework for the adoption and execution of 6GIIoE system.

Even though the focus was on the 6G-enabled IIoE system, literature related to 6G and IIoE priority areas, challenges, applications, and architectural framework strengthened through the literature review was utilized for reinforcing research and to help in the development of the theoretical framework. We utilized a strategic approach to explore literature relevant to $6 \mathrm{G}$, IoE, and IIoE, selecting those academic research works included in the literature review. The following steps were taken to determine relevant literature (i) using the key terms 6G, IoT, IoE, IIoE, Google Scholar (database-nature and timeliness of the topic), (ii) Google and Firefox was also used for industry white papers related to 6G, IoE, IIoE and various industrial applications, (iii) exploration of the leading journals such as IEEE, Science Direct (Elsevier), Emerald Insight was done, (iv) a search of academic research databases was pursued for relevant articles with the keywords IS, 5G, 6G, IoT IoE, IIoT, IIoE, theories, (iv) articles and conference proceedings listed in the references are related closely to the theme of the research paper, (v) summarized version of the articles and industry papers related to the theoretical framework and security/privacy matter of the topic. 
Table 1. Summary of the Literature Review.

\begin{tabular}{|c|c|c|c|}
\hline Reference & Publication Year & Research Direction & Main Focus \\
\hline Alsharif et al. [2] & 2020 & 6G Research Activities & 6G Networks \\
\hline CISCO [3] & 2020 & IoE & IoE Economy \\
\hline Singh et al. [5] & 2020 & IoE/ Blockchain & IoE/Fog Computing \\
\hline HUAWEI [8] & 2020 & Digital solutions & IoT \\
\hline Siemens [9] & 2020 & IoT $/ 2050$ & Industrial IoT \\
\hline Nord et al. [10] & 2019 & IoT/Theoretical Framework (TF) & Systematic Review \\
\hline Falkenberg et al. [12] & 1995 & Information System (IS) Theory & IS Limitation \\
\hline Aquilani et al. [13] & 2020 & Theoretical Framework (TF) & $\mathrm{TF}$ \\
\hline Giordani et al. [15] & 2020 & Few $6 \mathrm{G}$ use cases \& requirements & $6 \mathrm{G}$ \\
\hline Dang et al. [16] & 2020 & $6 \mathrm{G}$ Vision & Security \& Privacy \\
\hline Huang et al. [17] & 2020 & Survey Towards 6G & Wireless Evolution \\
\hline Gui et al. [18] & 2020 & 6G New Horizon & 6G Security \\
\hline Chowdhury et al. [19] & 2020 & 6G Applications & 6G Research Directions \\
\hline Mahmood et al. [20] & 2020 & 6G Networking & Machine Communications \\
\hline Janbi et al. [21] & 2020 & 6G/ Smarter IoE & $\mathrm{IoE} / 6 \mathrm{G} / \mathrm{AI}$ \\
\hline Sekaran et al. [22] & 2020 & $6 \mathrm{G} / \mathrm{IoT}$ & 6G/IoT Automation. \\
\hline Wang et al. [23] & 2020 & Industrial Control System/IIoT & IIoT \\
\hline Yang et al. [24] & 2019 & Potential 6G Techniques & 6G Techniques \\
\hline Saad et al. [25] & 2019 & 6G Vision \& Trends & 6G Services \\
\hline Clazzer et al. [26] & 2019 & IoT Applications in $6 \mathrm{G}$ proposed & 6G/IoT \\
\hline Tariq et al. [27] & 2019 & Speculative 6G study & $6 \mathrm{G}$ \\
\hline Zhang et al. [28] & 2019 & 6G/Super IoT & 6G/IoT Aspects \\
\hline Eppi Center [29] & 2019 & IoE & IoE \\
\hline Dai et al. [30] & 2019 & Blockchain/IoT & IoT \\
\hline
\end{tabular}

This review identified a substantial number of research publications that bestowed deep insights relevant to this research paper. Despite several research publications on $6 \mathrm{G}$, IoT/IoE, there is a severe scarcity of papers on 6G-enabled IIoE system researchbased studies. To our surprise, theory as related to 6G-enabled IIoE system adoption and execution is non-existent in the literature. We have outlined the review of the research papers as indicated by the citations below for various relevant topics and the selection of the literature criteria. A synthesis of our literature review identified that there were no papers considered by authors in the development of a theoretical framework on 6G enabled IIoE system adoption, execution, and recommendation research agenda. In the next section the study reflects on the exhaustive review of the recent literature on the 6GIIoE system including emerging 6G networking and technologies, existing IoT/IoE, information system (IS) discipline related to various theories as the theoretical foundation, sequential methodology, CIIW, and the contributions to the theories.

\subsection{Selection of Literature Criteria}

The exhaustive literature review for this study was done according to the following criteria:

- Well established databases (Scopus, IEEE Xplore, MDPI), peer-reviewed double blind reputable international journals and books from Emerald Insight, Elsevier, Wiley, Science Direct, MIT Technical Review publications, Nature, Springer, and Google, were the basis of this research.

- EPPI-Reviewer includes systematic reviews, meta-analyses, 'narrative' reviews and meta-ethnographies containing over a million items-version 4.11.5.2 (16 November 2020) [31].

- Peer-reviewed international journals and books published from the years 1980 to 2020 were consulted.

- Focus and Emphasis was given to recent books and journals to review and analyze the relevant information.

- The web search engine (Google Scholar) that indexes the full text scholarly articles.

- International Conferences proceedings. 
- $\quad$ PhD Thesis (Published Ones).

- Market and Scientific White Papers on connected intelligent industrial trends, perspectives, and applications.

\subsection{Essential Insights and Relationships between Relevant Topics}

A comprehensive review of the literature helped in identifying the key dimensions of the topics closely relevant to $6 \mathrm{GIIoE}$ systems' thinking. The literature review was divided into the following segments that bestows essential insights and the relationships between the relevant topics:

\subsubsection{Theoretical Framework Development/Information System (IS) Discipline}

Information systems (IS) are complex multi-dimensional phenomena [12] (p. 449) and signifies the use of various disciplines to study the development of theory based on the diversity of contexts within the relevant technologies of information. Although diversity in information systems practice, research has evolved and gained importance, but no well-accepted common theoretical basis for information system. Furthermore, there is no central corpus of a well-accepted theory.

Based on the content outlined in the sub-Section 1.2 to reaffirm our perspectives, the following scholars' points of view, as a validity, on the IS discipline are as follows: (i) Swanson [32] (p. 6) concludes that current theories describe little about IS innovation, (ii) Avgerou [32] (p. 2) contends that information system is a relatively unexplored subject, and (iii) Fichman [32] (p. 6) explains signs of exhaustion in the current research.

\subsubsection{GIIoE System}

1. 1.6G system requirements and technologies are going to bestow the following new theme: (i) novel man-machine interfaces; (ii) ubiquitous distributed computing and the edge (cloud) services; (iii) multi-sensory data fusion to create eXtended reality (XRaugmented reality-AR/mixed reality-MR/virtual reality-VR) [25] (p. 1) immersed experiences; and (iv) the physical world will be driven by precision sensing and actuated control. The IoE is an organic, complex, evolving ecosystem with limitless boundaries. 6GIIoE architectures in the literature is non-existent. This study argues for the support of 5-layer architecture for 6GIIoE ecosystem and the trends provides the formulation of design for the next generation infrastructure [6] (pp. 57063-57070).

2. CIIW is the fusion of $6 \mathrm{G}, \mathrm{IoE}$ and other emerging relevant technologies promises new possibilities, opportunities, services and immersive user experiences ubiquitously offering connected intelligent industrial applications such as connected intelligent factories (CIF), connected intelligent transportation (CIT), connected intelligent cities (CIC), connected intelligent robots and drones (CIRD), connected intelligent food and beverage (CIFB), connected intelligent retail (CIR) and other connected intelligent applications. Based on the $6 \mathrm{G}$ connectivity vision, as outlined below in the Section 5 , and with 6GIIoE applications, the CIIW is going to revolutionize endowing digitization towards personalization for every industry to provide enormous benefits.

\section{Theoretical Foundation and Approach}

\subsection{GIIoE Foundations and Boundaries}

Even though the $5 \mathrm{G}$ mobile system can support various IoT services, it may not be able to completely fulfill the requirements of IoE novel applications. Hence, 6G mobile systems are conceptualized to vanquish limitations of the $5 \mathrm{G}$ wireless system. IoE-based smart services raises the need for $6 \mathrm{G}$ wireless networks and comprises four pillars: data, things, people, and processes that are intelligently connected and describes a world where billions of objects have sensors to distinguish measurement and appraise their situation. IOE is also a concept that broadens the IoT prominence on machine-to-machine (M2M) communications to define an augmented complex system that also encircles people and processes connecting over private or public networks utilizing proprietary and/or standard 
protocols. IoE applications are categorized from sensor tools, smarter mobile devices, machine learning (ML) systems, interfaces used for remote appliances to machine learning systems to various types of distributed intelligent automated hardware. The Industrial Control Systems (ICS), which are closely related to people's lives, play a vital role in the development of the IIoE whose security affects the entire IIoE. ICS is connected to the Internet and unprotected in the cyberspace. To protect these precious assets, intrusion detection systems (IDS) have drawn much attention.

\subsubsection{Four Pillars of IOE}

Currently, IoT and 5G have started their honeymoon and are growing shinier applications such as remote surgery, smart factories, and unmanned vehicles. The $6 \mathrm{G}^{\prime} \mathrm{s}$ requirements will redefine the mobile system architecture and will empower use cases. Distinguishing that connectivity as the foundation, 6G will make future technological breakthroughs redefining the business models around data consumption because it will be not just a physical standard but also a virtual one providing new opportunities for novel networks services. In the quest to metamorphose industries for the digital era, IoE is one of the most significant components of ICT foundation.

IoE is the intelligent connection of people, process, data, and things with billions of objects to detect capacity and appraise their standing using various protocols. The IoE concept comprises of communication between machine to machine (M2M) communications, machine-to-people (M2P), and people to people (P2P) interactions. IoE is also regarding all the information collected from the four primary pillars (people, process, data, and things).

Without "people", everything else would be pointless and "people" themselves become the nodes on the Internet. "People", in the IoE, are to connect to the Internet in numerous ways through a myriad of devices or sensors. The evolution of is Internet toward the IoE, where everyone will be connected in relevant ways.

In the connected world of IoE, "process" plays a key role in how each of these pillarspeople, data, and things-interact with each other to deliver value for bestowing immersive user experience. With the exact process, connectivity becomes accordant and value is added due to the precise information being delivered to the right person at the right time in the appropriate manner.

The IoT is a vital pillar of the IoE. "Data" is still the foundation to be created, stored, then transmitted from one place to another and will continue to be at the epicenter of the internet paradigm. Currently, the number of new things connected to the Internet exceeds the number of new users connected to the Internet. Hence, we contend that the third generation "Internet for the people" became the fourth generation "Internet about things for the benefit of the people".

"Things" are composed of physical items like devices, sensors, and machines that are connected to each other and to the Internet. "Things" sense data evolve into being context-aware and bestow information to help machines and people in the IoE endowing relevant valuable decisions.

In summary, we have articulated about how the four pillars of IoE foster innovation in the new technology to empower user experience. As the internet progresses toward IoE, people will be connected in valuable ways. We believe that every challenge is an opportunity in dissimulation. Very often, huge opportunities emerge inscribing from colossal challenges. IoE will change industries and our daily lives. When the history of IoE is penned, its success or lack of success will be obstinate by: what is the benediction of IoE to humanity?

\subsubsection{G Challenges}

6G is not necessarily a single technology but a collection of mobile system technologies. A fundamental concept of the $6 \mathrm{G}$ future opportunity is the principle of 'New Services', from spectrum to infrastructure. Tiny cell network densification is the dominant theme of $6 \mathrm{G}$ wireless evolution. Densifying networks, while avoiding increasing inter-cell interference, 
is a key challenge. Additional key technical aspect of $6 \mathrm{G}$ networks will be the use of high-frequency spectrum, specifically the use of millimeter wave (mmW) spectrum with extreme low latency.

$6 \mathrm{G}$ cost modelling is a challenge that allows one to compare the difference between data traffic demand and network costs for different network scenarios. The costs of $6 \mathrm{G}$ infrastructure densification depend heavily on the required throughput density, periodic interest rate, and tiny highly dense base station infrastructure investment.

$6 \mathrm{G}$ considers the spatial rollout of telecommunications technologies over time. Often researchers focus on just one key aspect, such as modelling spatial viability. In mobile systems, there has been limited spatial modelling of the potential rollout of new technologies.

Network densification and additional spectrum will play a key role in delivering 6G, which will have a significant influence on the delivery economics. There has been limited modelling of both the spatial and temporal dynamics of the rollout for $6 \mathrm{G}$. While the current research on $6 \mathrm{G}$ networks provides insight into the vague technical specifications of various technologies, little emphasis has been placed on the rollout implications for different global infrastructure strategies.

\subsection{Discussion on Theories Related to Information Systems/IoT}

Corroborating the spasmodic research publications on theory advancement in IS/IoT discipline, some of the of research papers in this domain are the following:

2006 - Leidner and Kayworth promulgated a survey and review of theories on Information Technology and culture conflict [33].

2012-Evaluating and developing theories in the IS discipline was written with riche in content and published by Weber [34].

2016 - Wiener et al. developed an expanded theoretical framework for Information Systems Projects [35].

2018 - Chaudhuri and Cavoukian published a framework for IoT privacy [35].

2018-Hsu and Lin conducted a study examining factors seen as contributing to IoT service adoption using a research model based on the value-based adoption model [36].

\subsection{Relevant Theories Supporting 6GIIoE}

- The communication theory studies the process of information, interdisciplinary disciplines of interpersonal communications, psychological paradigm, philosophical and social dimension [37].

- IoT interface theory defines the interaction between the thing, service, and an accessor for which it is formalized as an interface automaton. For 6GIIoE applications, where "everything" and services interact with the physical world [38].

- The systems theory applied to 6GIIoE systems analysis applications. One of the vital mechanisms of systems analysis is systems thinking, enabling the contouring of systems from a broad perspective rather than specific events in the system [39].

- The Theory of Transparency where perpetual experience is often said to be transparent and be aware of the properties of the objects around us. This is an introspective fact. A transparency theory bridges the gap between them. The fundamental of transparency requires that the actions and decisions of industries be open to inquiry [40].

\section{Methodology}

Notwithstanding 6G still being nascent in its research and development, a quest to support 6G R\&D decision-making in government and industry has emerged. Despite significant technological and economic uncertainty, exploration of how the potential 6G rollout can be pursued both spatially and temporally is in policy formulation. Therefore, the coverage, cost, and rollout consequences of 6G networks globally are explored by extrapolating $5 \mathrm{G}$ characteristics. In this study, we focus on an appropriate methodology that covers $6 \mathrm{G}$ vision connectivity and its impact of annual capital intensity, infrastructure sharing and the end-user speed. Furthermore, varying annual capital intensity or 
deployment of a shared tiny high density cell network that can greatly influence the span of time to reach the $90 \%$ threshold benefiting ubiquitous connectivity globally is explored. Additionally, by integrating new and existing spectra, a 6G network capable of achieving Tera Bit speed with massive network capacity and extreme low latency is considered for universal service globally.

Based on the $6 \mathrm{G}$ challenges outlined in the sub-Section 3.1.2, the following are some of the research opportunities based on the sequential methodology that should be investigated: (i) cost modeling, (ii) ways to reduce the costs of 6G infrastructure rollout is via infrastructure sharing, (iii) spectrum and coverage obligations-frequency bands that are yet to be defined and allocated, therefore spectrum costs for 6G are unknown at present, (iv) sensitivity analysis to identify the cost boundaries which will have influence on $6 \mathrm{G}$ coverage and capacity, and (v) 6G policy implications.

A sequential methodology [41], Ref. [42] (p. 639) process enables the analysis team to concisely define the requirements, then planning for design prior to execution and ultimately focus on network densification and spectrum. A prospective study to evaluate 6GIIoE system is an integral part of developing and launching 6G network services and IIoE system technologies. In this study, we have proposed sequential approach to conduct analysis, design, implementation, and test to allow adaptations or modifications to aspects of a $6 \mathrm{GIIoE}$ system to validate the integrity of the trial. The application of sequential methodology in 6GIIoE system trials has the possibility of significantly improvement of the flexibility, and efficiency. To further advance the performance of 6GIIoE system trials, the sequential method can exploit novel adaptive approaches of various applications identifying the future directions in this field of research and development. The sequential methodology for the 6GIIoE system, as shown in Figure 1a,b, is adopted phase by phase: (i) the analysis, (ii) the design, (iii) implementation, and (iv) the testing.

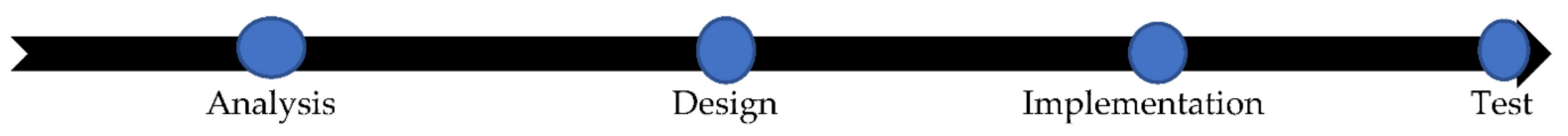

(a)

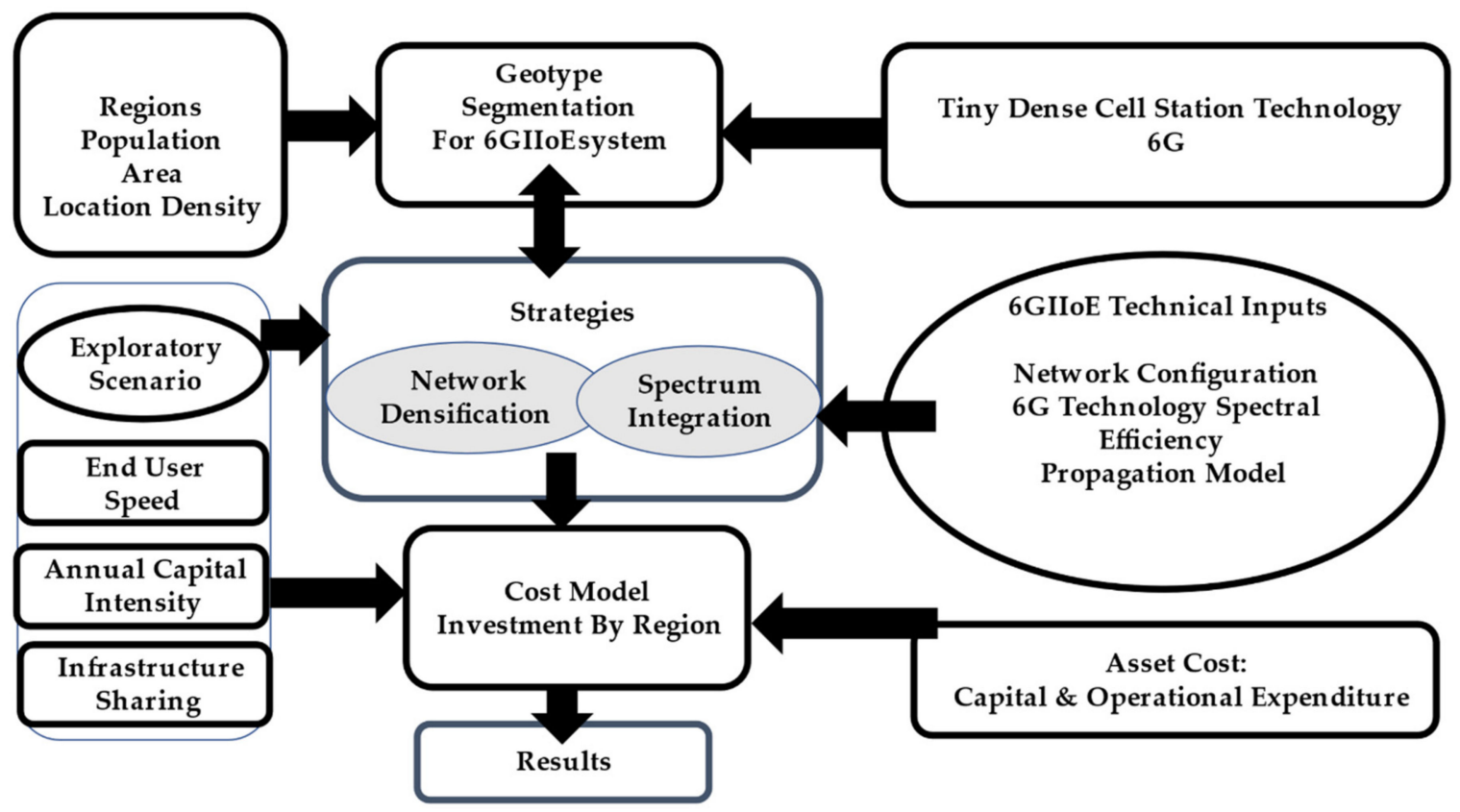

(b)

Figure 1. (a) Phase by phase sequential methodology process. (b) Sequential Methodology Process. 
Each part of the sequential methodology, step by step, is explained below to deal with the sources of uncertainty:

\subsection{Exploratory Scenario}

A set of exploratory scenarios should be utilized to demonstrate the temporal dynamics of the rollout and potential costs of research and deployment with respect to the $6 \mathrm{GIIoE}$ system. The exploratory scenario captures the uncertainty associated with the key technological, economic, and regulatory changes that focus on the relevant areas such as capital intensity, infrastructure sharing, and end-user speed.

\subsection{Strategies}

Two primary strategies need to be considered for network dimensioning and end user speed required in the exploratory scenario. The first option is to integrate spectrum into existing high-density tiny cells for achieving total geographic coverage in $6 \mathrm{G}$ to provide more bandwidth in areas of high demand. The second option is where network densification consists of 6G tiny high-density cell station rollout to be deployed using specific $\mathrm{THz}$ spectrum.

\subsection{Geotype Segmentation}

Depending on the geographical region, geotypes to be created to represent the key supply-side variables that affect rollout costs. Geotypes are classified based on population density as per the geographical region. The forecasted population coverage is derived from assumptions to interpolate the population coverage for a specific annual capital intensity.

\subsection{Infrastructure Sharing}

An overview of the cost model should be considered for a non-virtualized 6GIIoE infrastructure. The capital expenditure is considered based on annual capital intensity in relation to the pace of $6 \mathrm{GIIoE}$ system roll out. When $6 \mathrm{GIIoE}$ equipment costs are unknown, future generations of network equipment with enhanced performance tend to be a similar estimate in price to those of 4LTE advanced or 5G systems.

\subsection{Dimension of Networks}

Each exploratory scenario was to be done using a model to calculate the minimum number of tiny high-density cell stations required.

To attain the results, the rollout of a $6 \mathrm{GIIoE}$ system under business-as-usual conditions needs to be illustrated. Then exploration of the temporal dynamics under exploratory variables including annual capital intensity, degree of infrastructure sharing in deployments, and required end-user speed executed by using the cumulative population covered to represent the spatial dimension for a period.

\section{6GIIoE System Paradigm}

\subsection{G Connectivity Vision}

6G connectivity vision ought to be those needs that 5G cannot satisfy and can be defined with the following: (i) ubiquitous, (ii) intelligent, (iii) deep, and holographic. 6G mobile system [2] is expected to provide a massive coverage that will enable users to communicate ubiquitously with Tera bit per second speed that will re-shape the wireless communication evolution. 6G mobile systems will evolve to offer space/air/ground/sea integrated communication and the wireless tactile network as shown in Figure 2. 6G spectrum, technology, and features goal are shown in Table 2. 


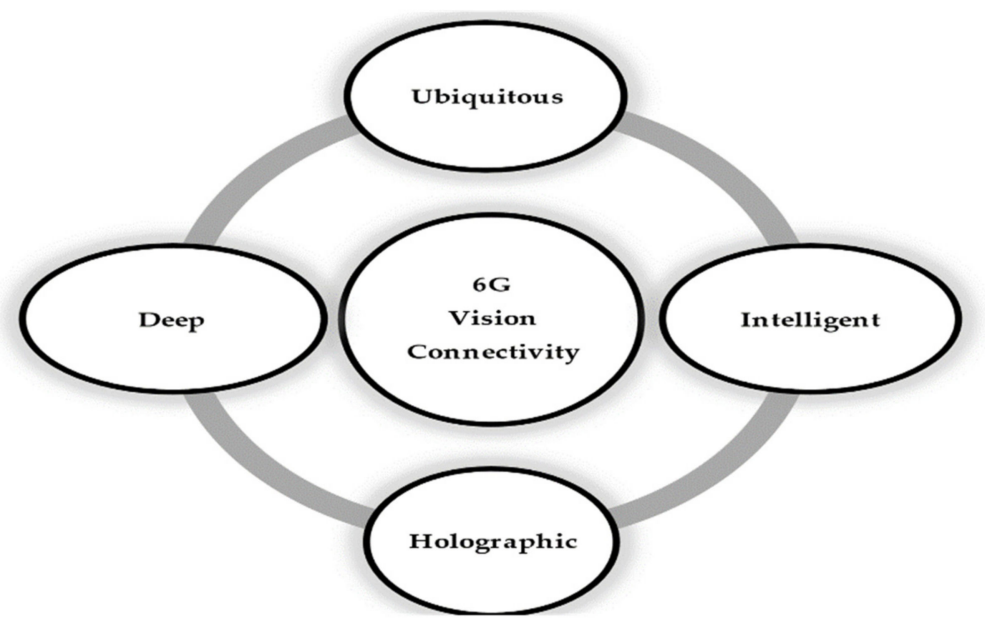

Figure 2. 6G Connectivity Vision.

Table 2. 6G Spectrum, Technology, and Features Goals.

\begin{tabular}{lll}
\hline \multicolumn{1}{c}{ Spectrum } & \multicolumn{1}{c}{ Technologies } & \multicolumn{1}{c}{ Features } \\
\hline Tera Hz & AI Based Wireless Communication & Space/Air/Ground/Sea \\
Visible Light Communication (VLC) & Advanced Signal Processing & Integrated Communication \\
Flexible Spectrum & Compressed Sensing & Wireless Tactile Network \\
(Free Full Duplex and Spectrum Sharing) & Very Large-Scale Antenna Processing for & \\
& THz, New Channel Coding. & \\
\hline
\end{tabular}

1. From deep coverage to "Deep connectivity" with the following characteristics: (i) Deep Sensing: Tactile Internet; (ii) Deep Learning/AI, and (iii) Deep Data Mining; Deep Mind: Telepathy, Mind-to-Mind Communication.

2. High fidelity AR/MR/VR will be ubiquitous, and holographic communication and display can also be carried out anytime and anywhere to enjoy a fully immersed holographic interactive experience. This communication vision of called "Holographic connectivity" with the following features: (i) holographic communication, (ii) high fidelity AR/MR/VR, and (iii) AR/MR/VR with seamless coverage anytime, anywhere.

3. The following are the characteristic of "Ubiquitous Connectivity": 3-dimensional coverage and connection, meaning the connection of anytime and anywhere, including Integration of Space-Air-Ground-Sea communication. Comparing "Deep Connectivity" and "Ubiquitous Connectivity" versus "Deep Connectivity" the latter emphasizes the depth of the connected object, while the former affirm the breadth of the distributed area.

4. "Intelligent Connectivity" is the nucleus of the 6G network, while "Deep Connectivity", "Holographic Connectivity" and "Ubiquitous Connectivity" establish the 6G network trunk.

The above four characteristics combined make the future 6G network's organic "soul".

\subsection{G New Service Classes}

$6 \mathrm{G}$ new services $[2,25]$, applications with performance indicators should be introduced to offer mobile Teraband reliable low latency connectivity (MTRLLC), massive ultra-reliable, low latency communications (mURLLC), human-centric services (HCS), and multi-purpose services (MPS) as shown in the Table 3. 
Table 3. 6G New Services, Performance Indicators and Applications.

\begin{tabular}{llc}
\hline \multicolumn{1}{c}{ 6G New Services } & \multicolumn{1}{c}{ Performance Indicators } & Applications \\
\hline MTRLLC & Rate Reliability Latency Requirements including Mobile & IoT/IoE/IIoT/IIoE \\
\hline mURLLC & Environment & XR (AR/MR/VR) \\
& $\begin{array}{l}\text { Energy Efficiency, Reliability \& Scalability } \\
\text { Massive Network Capacity }\end{array}$ & Legacy eMBB/URLLC \\
\hline \multirow{2}{*}{ HCS } & QoPE (Quality of Physical Experience) & wireless computer-brain interactions \\
& (WCBI) & Haptics, Telemedicine \& Mapping \\
\hline MPS & Stability, Sensing, Latency, Energy &
\end{tabular}

\subsection{IoE/IIoE Brief Overview}

CISCO [3] have used the term IoE and the foundation is built upon the "four pillars" of people, data, process, and things, as shown in the Figure 3. IoE extends industrial processes to enrich the lives of people and the intelligent connection of people, process, data, and things and describes a world where billions of objects have sensors to detect, measure and assess their status using standard and proprietary protocols.

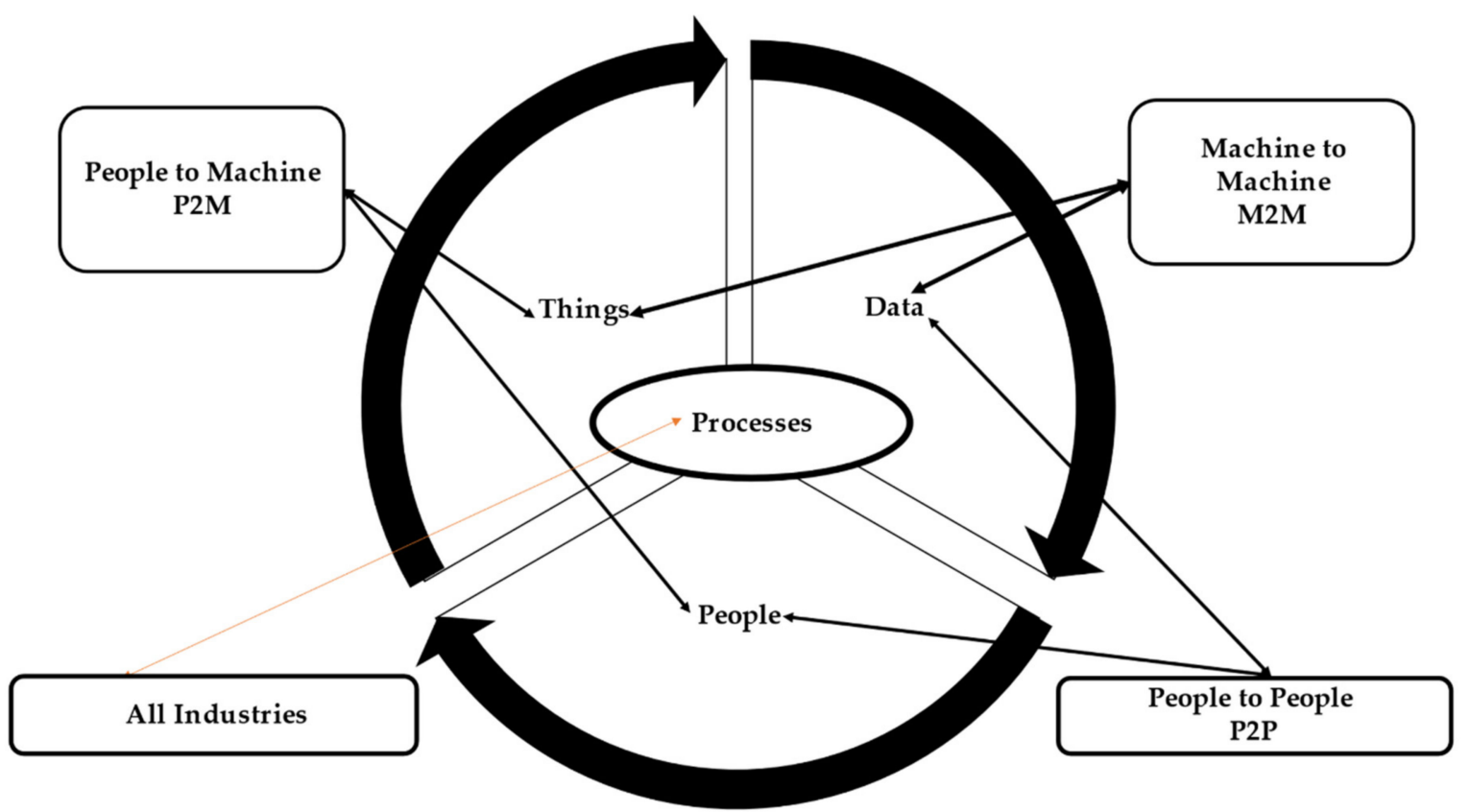

Figure 3. Industrial Internet of Everything.

Globally, people from different socio-economic backgrounds, religions and diversity using increasingly computing devices and the Internet because of the successful deployment of various innovative and killer applications based on IoE. This scenario has created a phenomenon of pervasive ubiquitous computing, universal usability or user-friendly interface design, and cross-cultural usability. Exploiting the cross-cultural universal usability and information system (IS) issues focusing on the world wide web (WWW) and mobile interactive dialogue using IoE becoming the trends open Innovations and requires further research in depth. This is a significant trend by users in how the 'IS' is utilized currently. Hence, the success of IoE/IIoE must consider the impact of cross-cultural usability by deep research requiring additional valuable research time and effort.

In this study, IIoE is defined: Smart devices or sensors are embedded into the intelligent systems for the product life cycle to build an information infrastructure empowering industry to expand their existing applications and even develop new ways to operate 
production. The enveloping of people, processes, data, and things is at the heart of IoE that creates the 'value' with respect to user experience. The IoT growth waves are leading to the eventual complete IoE. The advantages involve the use of IoE in supporting the attainment of socioeconomic and environmental goals. With each successive wave of added features and greater network connectedness, this leads to the IoE with many novel opportunities as well as risks. The $6 \mathrm{GIIoE}$ system has the potential to extract and analyze real-time massive data from the billions of devices or sensors or machines connected via 6G network and then to apply to aid all industrial applications. A study by CISCO predicted that $\$ 14.4$ trillion may be exploited within ten years (2013-2023) by implementing IoE with M2M, M2P and P2P [3].

IIoE projects involve propagation of sensors, devices, complex processing events, and APIs. Project implementers overinflate the built-in integration of IoE platforms without due deliberation to the intricacies involved. The authors suggest the following strategic areas for industries to customize their mobile re-architecture: (i) Mobile App development services; (ii) Social Media Integration; (iii) IoE Integration Services. IIoE projects are complex and are emerging fast to become a popular technology for all industries.

Concurrently, intelligent offices are seeking to leverage smooth and streamlined operations for all industries. Predictive servicing is becoming the gold standard in customer service, therefore IIoE remote monitoring for the predictive servicing provides peace of mind and a meaningful value proposition:

- Sensors need to be installed at the customer's premises to be monitored visually via relevant applications.

- Publication of user data to the Cloud to build an overall picture of the user's consumption habits, and trends.

- Remote monitoring app to alert customers when the consumable falls below a set level and improve customer experience.

- IIoE remote monitoring with the consumables supplier's ERP (Enterprise Resource Planning) system for scheduled delivery.

- Specialize the situation for each specific industrial application, product access and purchase.

\subsection{IIoE Trend: Massive Data Analytics and Predictive Maintenance}

IoE sensors, devices and endpoints are growing exponentially in size, form, function and "massive data" remains the foundation and mettle of IoE applications and software. This scenario empowers to identify novel ways of improving and leveraging the interoperability of people, data, processes, and assets. Looking into the future, by 2030 over hundreds of trillions of gigabytes of data will be generated by IIoE that enables all types of applications including deep learning of manufacturing operations globally. Massive data analytics (MDA) is a growing trend phenomenon. IIoE analytics of massive data is fundamentally different from current data management paradigms to assemble the massive data in a unique manner providing meaningful useful insights and optimization of the connected intelligent factory (CIF) operation. The efficiency of an IIoE system efficiency grows if data analysists can analyze in a timely fashion the incoming data and operationalize those insights, and massive data sharing concepts among competitors will be one of the ideal ways to make sense of this massive volume of information. With the disposition of AI and/or machine learning (ML)-based predictive maintenance capabilities, remote massive data collection IIoE platforms via 6G communications can quickly update machinery and devices enabling minimizing down time substantially and reducing cost significantly.

\subsection{Role of $6 \mathrm{G}$ Revolutionizing IIoE}

6G will become the "eyes and ears" of all IIoE systems since it provides real-time massive data collection and analysis. The pivotal role of 6G communications in IIoE, referred to as 6GIIoEsystem, will empower and precipitate fifth industrial revolution mission and offers the following advantages to create significant value: 
- The extremely low latency, massive network capacity, high-speed (>100 Gbps) for massive data exchange hastens the realization of digitization towards personalization.

- Enables reducing operation time due to extremely low latency and increase in massive data throughput leading to enhance process productivity with quality control and leverage optimization process for expanded connected intelligent automation.

- Can attenuate the complexity of supply chain networks, and warehouse management, helping businesses to efficiently operate in dynamic industrial environments.

- Endows the next era of AI driven enterprises powered by 6G connected edge (cloud) computing percussing into massive volume of data to unleash insights that would otherwise not be attainable.

- Entrusting the sustainability of all industries managing the vast volume traffic to hyper e-commerce by enhancing $6 \mathrm{G}$ network accessibility at a faster pace accelerating online purchases, order placements, customer delivery and have the potential to disrupt on-site job functions through virtual meetings as well as remote working environment.

\subsection{Why 6GIIoE Is So Appealing?}

An unprecedented proliferation of new IoE services for industrial applications are emerging such as extended reality (XR) services (augmented, mixed, and virtual reality (AR/MR/VR)) to telemedicine, flying vehicles, wireless computer-brain interfaces (WCBI), and connected intelligent systems for all industries. These emerging IIoE services will need computing functionalities and end-to-end co-design of communication which has been overlooked. To exploit these unique challenges for the new breed of services require massive bandwidth frequencies beyond sub- $6 \mathrm{GHz}$ to transform wireless systems to meet the requisite IIoE scenario. To overcome these future challenges to catalyze the deployment of new IoE services require $6 \mathrm{G}$ ) wireless system. The drivers of $6 \mathrm{G}$ will be a convergence of past trends and emerging trends that include new services and the recent revolution devices such as smart wearables, implants, AI, 3D environmental mapping and XR devices.

There is a growing need to connect exponentially more devices, more sensors and install things like real-time cameras to perform massive data analytics (MDA). It is becoming vitally important to achieve better reliability, greater scalability, performance, easier accessibility, broader reach with a lower cost of ownership and need to increase connectivity with ultra-low latency. 6G is an ideal solution and can make a real difference for industries seeking augmented reality (AR) and virtual reality (VR) to help operators service equipment. To interact with robots is change and there is a need for real-time video to do spatial analytics for man and machines interactions to unlock a new level of intelligence with a new layer of connectivity with $6 \mathrm{G}$ that helps drive better business outcomes. 6GIIoE is a realm that will dramatically shape the manufacturing landscape, the way factories operate, and workforce needs. While the following financial rewarding possibilities of CIF certainly appeal to the dreamers and innovators, the clear payoff ensures that it is a pragmatic path that industry decision-makers will keenly follow:

- Maximizes revenue growth.

- Reduces operating cost.

- Increases asset efficiency.

\section{6GIIoE Architectural Framework}

Based on the literature review standards, specifications, and reference architectures, (specifically) of 6G-enabled IIoE system is non-existent. Scholars are working diligently to develop 6G standards and specifications. We are of the opinion that global 6G standards and specification by the ITU (International Telecommunication Union) will emerge prior to the year 2030. 6G communication architecture should enable IIoE devices, sensors, machines to not only connect to the Internet but also to communicate with one another autonomously. The current literature describes several $6 \mathrm{G}$ and IoT/IoE architectures $[43,44]$ (p. 134010). In contrast to the 3 and 4-layers architectures nevertheless, there is no proposed global 6G and IIoE standard architecture yet and drawing from the authors' extensive 
experience in 5G-enabled IoT/IoE architecture design as well as from the literature review of relevant research work, we believe multiple layers are essential to capture the complexity of the 6GIIoE system architecture. Hence, we have proposed and discussed below a simplified 5-layer 6GIIoE modular architecture as shown in Figures 4 and 5:

- The perception layer comprises the physical components: all sensors, devices, and machines.

- The transmission layer includes the information communication.

- The middleware layer consists of communication protocols, 6G, Wi-Fi, edge (cloud) computing and software to analyze data.

- The application layer deals with IIoE intelligent applications.

- The business layer defines the business model.

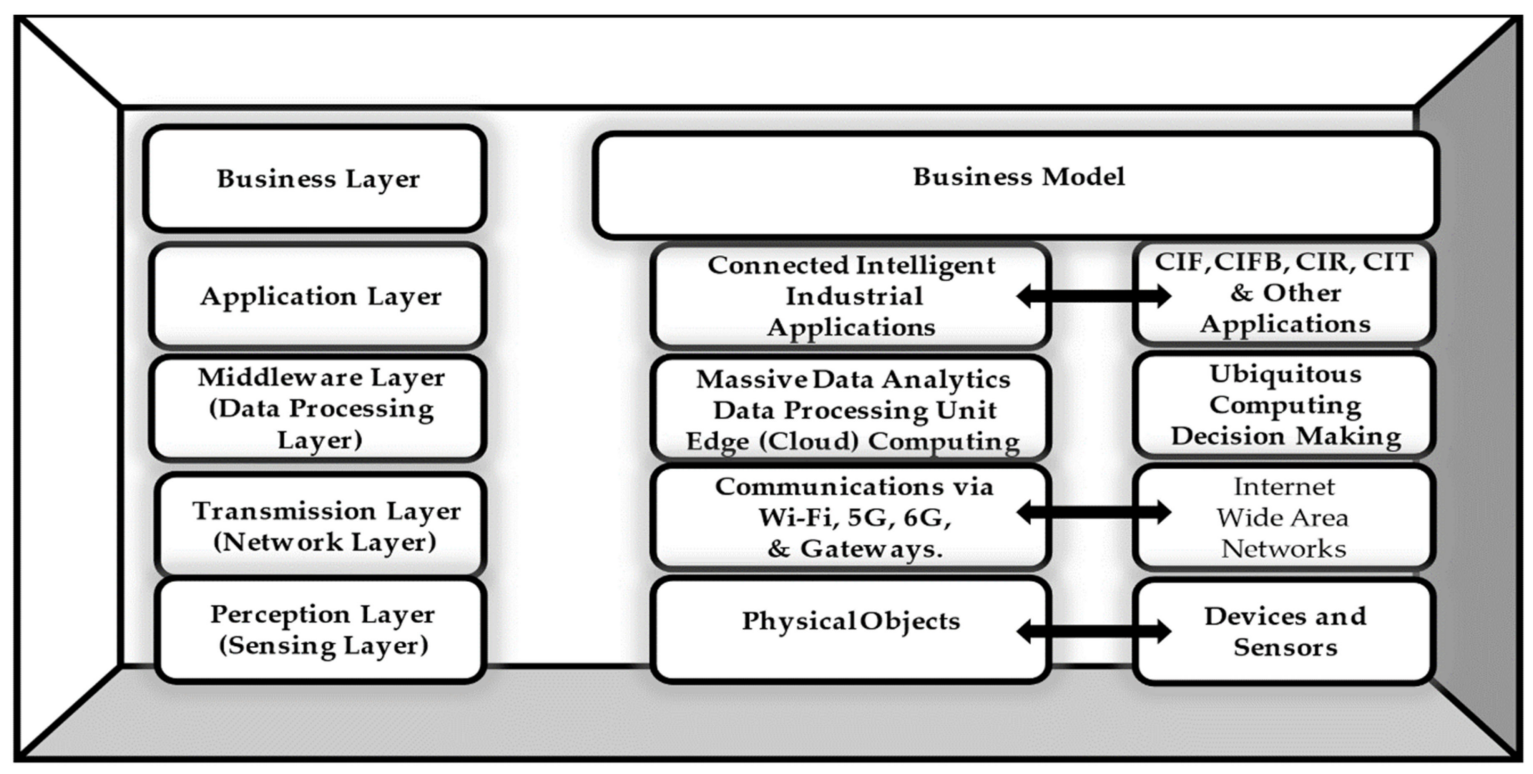

Figure 4. Five Layer 6GIIoE Architectural Framework.

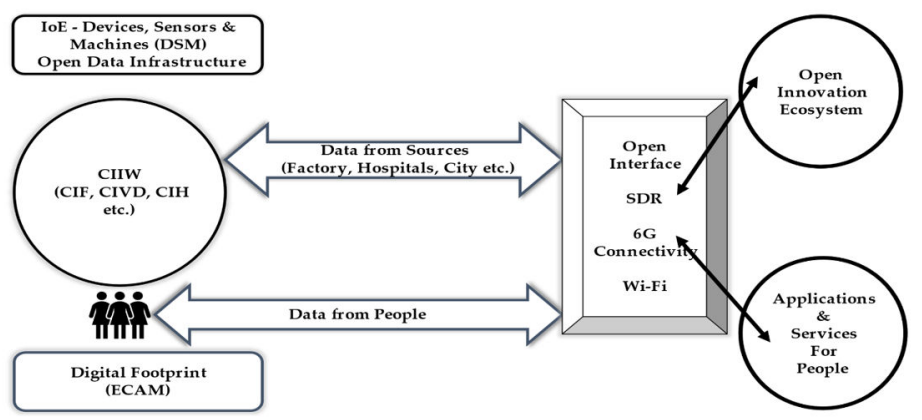

Figure 5. 6GIIoE System Architectural Framework.

The operating system (OS) is the virtual application platform that aggregates open innovations for various industrial applications and people (e.g., Apps can be developed using intelligence from the Digital Edge-Cloud on which it operates) and should serve as the 'operations center' for the public services domain. The Digital Edge-Cloud (DEC) [44] denotes the edge-cloud platform (with embedded hardware and software) that aggregates intelligence spanning multiple use cases, and more widely across IIoE applications, namely CIIW etc. that could benefit from composite information.

We cite one use case example: smart cities with 6GIIoE system. We propose significant pilot efforts around the globe that can be applied for Smart Cities with 6GIIoE 
System. 'Realizing the vision of smart cities in the USA' initiatives can be focused on environmental aspects with underlying IoE technologies beyond Edge-Cloud-AnalyticsMobile (ECAM). Having a universal digital footprint for various IoE applications could help tap into the enormous benefits. A wireless communications infrastructure, such as 6G, is built on fiber-optic and/or Wi-Fi (6G-based) backbone network to serve the platform that moves massive data from devices/sensors/machines (DSM) for aggregation in the DEC. DSM infrastructure can be formed of existing physical infrastructure equipped with wired/wireless P2P, P2M and M2M devices/sensors/machines to enable detection and notification of events to the higher layers in the 6GIIoE architecture. Our view is that ECAM (Edge/Cloud/Analytics/Mobility) technologies enable powering up the 'Digital Footprint' in the 6GIIoE system architectural framework, as shown in the Figures 4 and 5, though the complexities of a large-scale CIIW deployment are yet to be understood. The major challenge would be in identifying scalable, cost-effective, and interoperable technology solutions to build the device/sensor/machine (DSM) infrastructure ensuring raw / filtered massive data flows through to the 'Digital Footprint'. Considerable R\& D work needs to be pursued to actualize the operations of various connected intelligent industrial applications.

Previously security involved a simplistic approach followed the layered architecture for the IoE. This ignores both the complexity of the $6 \mathrm{GIIoE}$ system and the need to provide security functionality in different places and in different forms. For example, authentication is needed both for applications and all individual devices. Therefore, in this study, a new model is proposed which is layered in vertical and horizontal planes to inter-operate. The horizontal planes cover the architectural components of the 6GIIoE system, from devices to service composition up through applications to the end-user. The vertical planes cover security services required at various system architecture levels and mechanisms.

The proposed 6GIIoE architecture is composed of many elements from gateways, sensors, device management, connectivity, and application platforms. It is also important to understand that the functional components of a $6 \mathrm{GIIoE}$ architecture that encapsulate the diverse security requirements and issues, therefore, a five-layer, three-dimensional security architecture is essential. It becomes daunting for an enterprise to take the initiative to construct the $6 \mathrm{GIIoE}$ architecture at the outset with these elements. For CIIW, the infrastructure layer requires the following technology, unique characteristics and challenges that should be addressed effectively, as described below:

- interoperable that keeps up with the industry's dynamic needs. Sensor/Machine Infrastructure Layer-Technology, Characteristics, and Challenges:

- Inexpensive, light weight, miniaturized IoE—devices, sensors, and machines with almost no physical security and memory capability.

- $6 \mathrm{G}$ network connectivity with low power communication protocols.

- Support traditional security algorithms.

- Embedded in physical structures so that wireless devices that operate autonomously with secure remote management.

- Ultra-low power circuits and communications.

- Devices/sensors/machines that can run on a single battery.

- Ambient and regenerative energy harvesting capabilities.

- Adaptive to real-time analysis of sensor/device/machine massive data.

As the 6GIIoE ecosystem continues to evolve, the following trends should be considered formulating architecture and design for the infrastructure.

\subsection{Prototyping Hardware}

For the industrial applications, the challenge of 6GIIoE hardware architecture and design depends on the confounding array of use case requirements. Example: Taking sensor temperature. Based on the criteria or requirements like temperature range, stability, response time and accuracy, the selection of sensors from hundreds available is key. A typical wireless sensor may not be available that fully meets the needs. That is where 6GIIoE hardware rapid prototyping helps. Hardware prototyping enables to build a 
customized hardware prototype in a short span. A ready-to-use portfolio, 6Gwireless modules, compatible sensor, interface, and compilers as well as prototype boards can create the optimal hardware mix-and-match that may match with specific industrial use case. Through quick hardware prototyping, enterprises can ratify the 6GIIoE technical and business viability of their target solution in a cost-effective and agile manner creating a foundation for a successful outcome.

\subsection{Wireless Connectivity}

Generally, a factory uses and operates with legacy industrial systems that are being rarely connected by wide area networks or latest wireless communications such as 5G or emerging 6G. Furthermore, the legacy industrial systems employ proprietary communication protocols for automation purposes and the lack of interoperability among these protocols hampers the execution of a monitoring and control network for the entire operations of the factory. Typically, data is captive within discrete control loops generating numerous data silos on the manufacturing floor.

\subsection{Software-Defined Radio}

At present many industrial facilities have implemented wired networks (LAN) and wireless networks such as Wi-Fi. A legacy IIoT architecture incorporates multiple radio protocols and standards. Most industries are exploring the deployment of new types of connectivity such as 5G or the emerging 6G to exploit high-value use cases. Hence, it is important to create backward compatible and an efficient 6GIIoE architecture that can accommodate software-defined radio (SDR)—a radio communication [44] (p. 134011) method where most signal processing is done using software. IIoE gateways leveraging SDR or 6G can implement and decode different protocols to save infrastructure cost and complexity. With 6G wireless connectivity, the above architecture can achieve simple software updates offering the possibility to enterprises to dynamically adapt to future operational and technological changes.

\subsection{Portable Container-Based Platform Design}

A portable, container-based design 6GIIoE platform provides industrial users with full flexibility and enables easy migration to another server as required without compromising the functionality of the application. Customized applications can be packaged and delivered in a portable standardized container. Users can decide which specific platform functions and applications with the modular architecture.

\section{6GIIoE Priority Areas, Applications, and Challenges}

\subsection{Priority Areas}

Based on the exhaustive literature review, survey (mentioned in the Section 3.2) [33] and white paper published by Siemens, Huawei, Cisco [3,8,9], findings divulged that IoT/IoE affects various industries, and the topmost priority areas are customer experience, asset management and financial decision making.

First on the list of priority area is to enhance customer experience. The following ways 6G enabled IIoE system can enhance the customer experience:

- $\quad$ Perpetual monitoring of customer experience with company offerings.

- Personalizing the situation for each customer.

- Updating products and services based on learning over time with customer interactions.

- Continuous innovation product access and purchasing experience.

The second and third priority areas are asset management and financial decision making, respectively. The 6G enabled IIoE system can play a significant role in financial decision making by facilitating asset tracking and managing real time visibility. Top priority areas may differ based on industrial applications. Customer experience and financial decisions (asset management tracking) facilitate a sound foundation of functional areas for practitioners and academic researchers to appraise. 
Furthermore, the following specific priority areas of $6 \mathrm{G}$ and IIoE needed attention:

- 6G should entrust MTRLLC for mobility management at high frequency mmWave bands and $\mathrm{THz}$ beyond.

- $6 \mathrm{G}$ requires a move towards radio centric system design and end-to-end co-design 3CLS [25] (p. 3) (Convergence of Communications, Computing, Control, Localization, and Sensing) driven by artificial intelligence (AI).

- $6 \mathrm{G}$ vision should be driven by a diverse portfolio of applications, technologies, and techniques.

- 6G high density tiny cell base station paradigm should embark upon a new era of smart surfaces communicating with human-embedded implants.

- Performance target analysis and optimization of 6G should operate in 3D space.

- IIoE: customer focused perspectives-the term "IIoE" is about the ways IoE can be used for industrial applications. IIoE focuses on improving connectivity between devices, time/cost savings and achieving efficiency optimization for industries. IIoE is amid a fundamental transformation with connected devices growing, up to 75 billion by 2025 [45], more and more enterprises are entering the industrial IoE arena. In the past, IoE interconnectivity was exclusively for enterprises that had the resources to build an IoE system. Nowadays, companies opt for a pre-built and full-stack managed IoE service that can offer the necessary hardware, software, and edge (cloud) infrastructure to handle billions of concurrent device or sensors, or machine connections to edge (cloud) network infrastructure such as 6G.

- IIoE: predictive maintenance and analytics (PMA)—Industries are using IoE-enabled systems to send out notifications to maintenance crews to predict when equipment is about to fail. This reduces the amount of maintenance time and allows maintenance crews to spend time to focus on other tasks. IoE-enabled systems collect massive data on how often these notification failures are sent out. Industries can use and communicate this massive data via $6 \mathrm{G}$ to create maintenance timelines and save significant cost and time, allowing teams to service equipment before it even fails. Predictive maintenance and analytics (PMA) [46] are becoming one of the most important use cases for industrial analytics.

\subsection{Applications}

The motivation of this section is to discuss various industrial application areas of IoE. Numerous industrial applications and services can be employed via 6GIIoE system. Here we briefly outline some of the example application areas. The CIIW comprises of industries related to transportation, vehicles, offices, buildings, manufacturing (factories), retail stores, E-commerce, food and beverage, health, port/harbor, financial services, banks, insurance entities, logistics/supply chains, energy sector and other applications becoming priorities areas to be connected intelligently. The CIIW is going to be a pervasive phenomenon in which objects (devices, sensors, machines, and wearables) can process, store, and exchange information seamlessly using IoE technologies seeking to be intelligently connected. Below are some of the 6GIIoE applications in the CIIW that can leverage the growth opportunities [8] (p. 3), [9,10] (p. 102), [11]:

- $\quad \mathrm{CIF}-$ Connected Intelligent Factories.

- $\quad$ CIFB - Connected Intelligent Food \& Beverage.

- CIFSBI-Connected Intelligent Financial Services, Banks, and Insurance.

- CIT-Connected Intelligent Transportation.

- $\quad$ IC-Connected Intelligent City.

- CIE—Connected Intelligent Energy system.

- CIR-Connected Intelligent Retail

- $\quad \mathrm{CIH}-$ Connected Intelligent Healthcare

- $\mathrm{CIHP}$-Connected Intelligent Harbor and Port.

This study specifically describes two specific applications (CIF and CIFB) related to connected intelligent industrial world (CIIW): 
- CIF-connected intelligent factories: At present, factories are connected by wired connections with fieldbus or industrial Ethernet, for connecting equipment to control systems to empower the factories with a secure and structured way for massive data access and control. 6G can target factory automation with extremely low latency communication with massive network capacity realizing the mission-critical applications in an industrial environment enabling devices or sensors to "offload" some processing capacity to the network. The IIoE needs a variety of technologies complementing each other to deliver the enhancements in agility, efficiency, and automation and requires real-time streaming for actionable intelligence, machine learning capabilities for identifying patterns and predicting behavior, $\mathrm{XR}$ (AR/MR/VR) to complement manual guidance and training. The 6GIIoE factory platform [22] (p. 143460), [47] (pp. 2-4) analytics software can achieve the following benefits for many industries: (i) makes IoE simple and easy to deploy without writing code, (ii) connects and manages any asset and analyzes any amount of data automatically in real time, turning data into actionable insights to reduce costs and improve efficiencies to build innovative and differentiating applications, and (iii) continuously improve production processes benefiting to visualize analytics for faster decisions, analyzing to solve problems, and monitoring operational performance. The $6 \mathrm{GIIoE}$ factory platform, as shown in the Figure 7, is an edge (cloud) platform using open-source-software via application programming interface (API) in the automotive industry. It stores massive data collected via data connection interface (DCI) from a variety of equipment's and autonomous logistic systems in a single cloud network. Thus, the 6GIIoE system realm will dramatically shape the 21st century manufacturing landscape transforming the way factories operate, and workforce needs. While the following financial rewarding possibilities of CIF appeals to the dreamers and innovators, the clear payoff ensures that it is a pragmatic path for industry decision-makers to follow keenly: (i) maximizes revenue growth, (ii) reduces operating cost, and (iii) increases asset efficiency. The development of a 6GIIoE factory platform should include the following: (i) the ability to develop a software platform (in-house and keep improving as well as evolving the platform even after the software platform has been put into operation, (ii) enhancing the firm's ability to leverage KLAS (Kanban, Lean, Agile and Scrum) [48-51] development techniques, (iii) acquiescing to share massive amount of data with partners as an open source platform to develop and enhance applications linking various devices to deliver various products and to notify the operator of equipment abnormalities contributing to the realization of a safe and secure system, and (iv) software engineers of an industrial firm can work swiftly and collaboratively by hastening improvements in 6G, ICT, OT, and IoE technologies attaining efficiency, quality, and cost savings.

- CIFB-connected intelligent food and beverage: The traction of food consumption is exponential globally as countries develop food and beverage supply chains and increase throughput to meet the demand. The primary aspect of empowering IoE (availability of 5G/6G connectivity, processing and cost of devices or sensors) have reached at a point to wide scale execution of IoE solutions across the food and beverage (F \& B) value chain [22] (p. 143461). One key IoE use case for the F \& B industry is advance tracking - cargo level tracking to complement asset level tracking by filling in the massive critical data visibility gaps at each pallet level and enabling the entire supply chain visibility and managing risk for the F\& B stakeholders. The Internet of F \& B can facilitate an unparalleled level of data visibility and traceability for all the stakeholders of the F \& B industry leveraging 6GIIoE system technologies in connectivity and security as shown in Figure 6. 


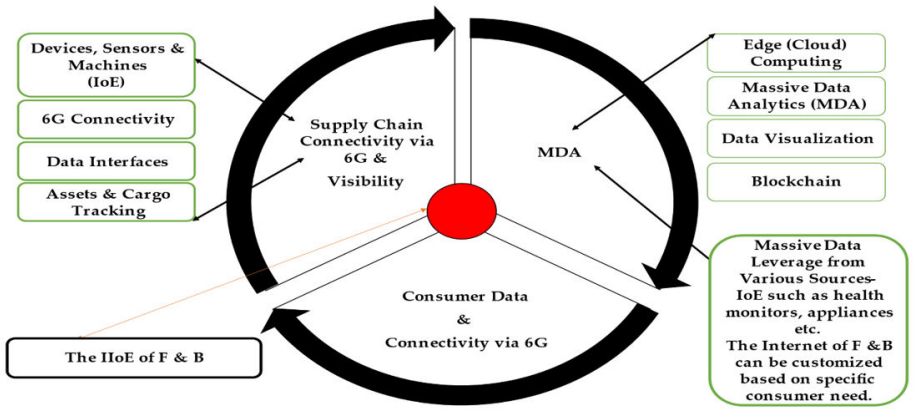

Figure 6. The 6GIIoE System for Food \& Beverage-The future of Food and Beverage Traceability.

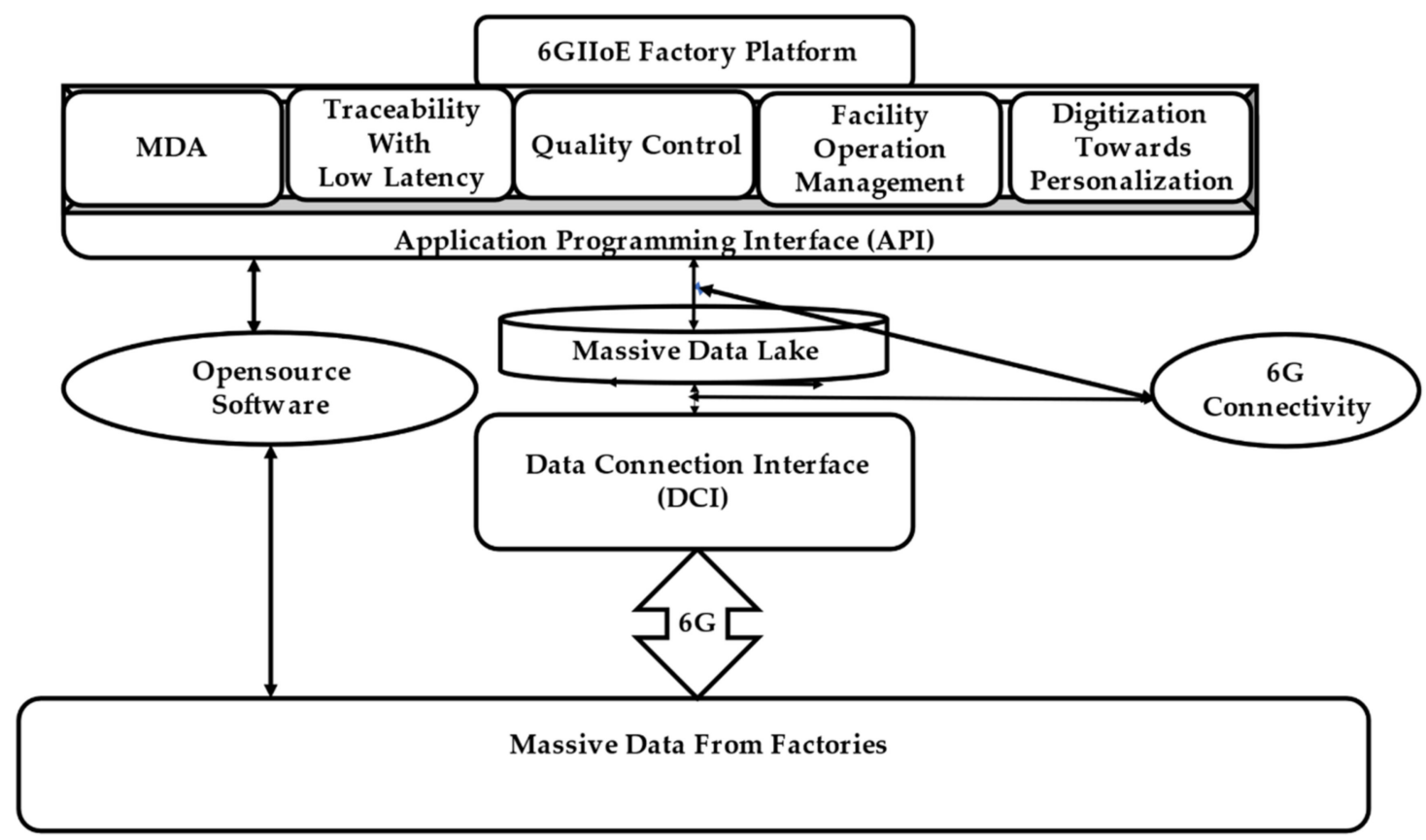

Figure 7. Connected Intelligent Factories.

\subsection{Challenges}

Every generation of technology approbation and execution has its daunting challenges. 6G-enabled IIoE system-based solutions involve many technologies, generating a complex environment. The following are the challenges [22]:

- Integration of new technology solutions into prevailing technologies.

- Management of a complex system

- 6 G Networking issues, specifically cloud/edge data analytics and management.

- Security and privacy

6G-enabled IIoE system services is perceived as an integral part of most industries' future requirements in a ubiquitous manner. The privacy, security and trust are of utmost concerns, specifically when 6G-enabled IIoE technologies dependent on sensors, devices, machines, wireless communication networks, cloud storage, and the relevant software solutions. As per the survey of the USA technology leaders, the topmost obstacle to IoE growth are privacy and security worry. Furthermore, the following are the primary security challenges IoT: mobile system security, authentication, trust, secured software, and privacy. We suggest the following standards to relevant stakeholders for the ways to address the IoE security issues:

- Security measures must be given priority attention.

- At the design phase the security issues need to be addressed. 
- Proven security practices need to be adopted during the building phase.

- Transparency is vital across all phases of IoE adoption and execution.

While the advantages of $6 \mathrm{G}$ enabled IIoE services are irrefutable, security and privacy aspects are not keeping pace with technology innovation. Hence the authors contend that 6GIIoE system security and privacy concerns must be taken care of prior to user trust in offering the applications. Industries face daunting challenges [22] (pp. 143455-143459) to balance the benefits of new technology and the risk associated with data breaches. Furthermore, the pressure to keep everything "intelligent" is increasing. IIoE can provide great benefits only when smart machinery is reliable. While there are many IIoE technical challenges, such as scaling the solution, it is important to focus on the two aspects: (i) how to generate recurring revenue stream and profit, and (ii) how to find a product-market fit. Essentially, the value proposition and the business model. While value propositions are the benefits for the IIoT system, a compelling business model ought to demonstrate to attain that value to market.

Additional challenges to adopt intelligent infrastructure for the 6GIIoE systems deployment include the following:

- Connectivity outage - constant need for uninterrupted connectivity if an enterprise

- Reliability in performance and delivering value to the customer.

- Data storage, and data management performance.

- Processing capacity and maintaining visibility of "things".

- Building legacy and IIoE infrastructure.

- Moving traditional OT to the edge of the network.

- Selecting the right tools.

- Accessibility anytime and anywhere,

- Interoperability in a heterogeneous environment.

\section{Towards a Novel Theoretical Framework}

The literature review shows that there have been few theories developed in IoT domain. This is the first time an integrated 6G-enabled IIoE system of challenges, priority and application areas in an enactment and execution of novel theoretical framework was developed. The primary contribution of our research work is the creation of a novel theoretical framework on the $6 \mathrm{GIIoE}$ system approbation and execution which is nonexistent in the literature. In our view, a theory makes a novel contribution to a discipline in the following ways:

- A theory's nucleus phenomenon might not have been covered by previous theories.

- A theory's originality might emerge due to essential changes it causes to extant theory outlining the bounds of the theory concisely.

- A theory could be acknowledged as novel due to the fact that its construct bestows well-known central phenomenon in new ways.

The theoretical framework developed in this paper falls under a theory's focal phenomena that has not been covered by prior theories.

The proposed novel theoretical framework is based on a set of relevant theories, models, concepts, 6GIIoE paradigm mentioned above in the Sections 3-7. The developed theoretical framework is classified into cyberspace and physical space and has the following aspects, (i) technology innovation and adoption of 6GIIoE, (ii) 6GIIoE stakeholders-as people influenced by the interest in the 6GIIoE system, (iii) usage consideration of 6GIIoE priority areas, challenges, specifically privacy and security, and (iv) usage change as people gain confidence and trust in the 6GIIoE system.

It is imperative to understand the enabling technologies through the intervention of 6G and IIoE from a virtual environment to every day live environment. In this context, 6G and IIoE represents the actual link between virtual space and physical space [13] (pp. 1112), specifically it functions as cyber-physical systems (CPS) domain. CPS is an evolution of cyberspace that provides the IIoE objects positioned at the epicenter of the framework 
as a link between physical space and cyberspace. Hence, 6GIIoE system offers one of the central enabling technologies of this theoretical framework.

The 6GIIoE priority areas will vary from industry to industry depending upon customer experience, asset management, and financial decision making. Stakeholders (partnerships) play a vital role in the formulation of a theory; therefore, researchers need to pay attention and decide which stakeholders (partnerships) should be included in a theory. Most theoretical frameworks in the IS discipline are process frameworks or variance [52]. Scholars suggest that theoretical development should be from a process perspective due to the technology adoption. 6GIIoE challenges (security, privacy, confidence, trust) are considered as an important aspect of this study, hence security measures are paramount to attain confidence and trust in the 6G-enabled IIoE services. Confidence creation and conviction management plays a prominent role in the 6GIIoE system for reliable data fusion, improved customer privacy, services with context-aware intelligence, and information security [10] (p. 103). Additionally, in this study, additional contributions, based on the extensive literature [10] (p. 103), on confidence and conviction management was established for the development of a $6 \mathrm{GIIoE}$ system theoretical framework. In our view, confidence and conviction creation and sustainability ought to be a proactive approach in all phases of 6GIIoE system adoption and execution. Furthermore, scholars have conducted extensive surveys of trust analysis and computation models for IoT concluding with guidance for trust computation research. Based on such prior surveys and the insights, this study gave credence using those survey judgements while developing the novel theoretical framework. While confidence and conviction management, security and privacy are essential to the 6GIIoE system adoption and execution success, privacy and security are the harbinger to the creation and sustainability of trust management [10] (p. 103). Thus, the above insights regarding the developed framework help to investigate 6G-enabled IIoE system.

The development of the proposed theoretical framework is also focused on the building blocks and practices as shown in the Figure 8 are: (i) the design of 6GIIoE architecture to exploit the priorities, challenges, and opportunities, (ii) cooperation and participation among the stakeholders in the entire 6GIIoE value chain, (iii) interactive actions between the 6G, and IIoE applications to achieve optimum performance in CIIW. Based on the building blocks mentioned below, the 6GIIoEtheoretical framework is constructed and the framework embraces the stakeholders' interactive work that includes 6G, IIoE applications. This framework can be used as an education model and guide for all industry stakeholders including suppliers, providers, customers, and decision makers who are seeking $6 \mathrm{GIIoE}$ systems.

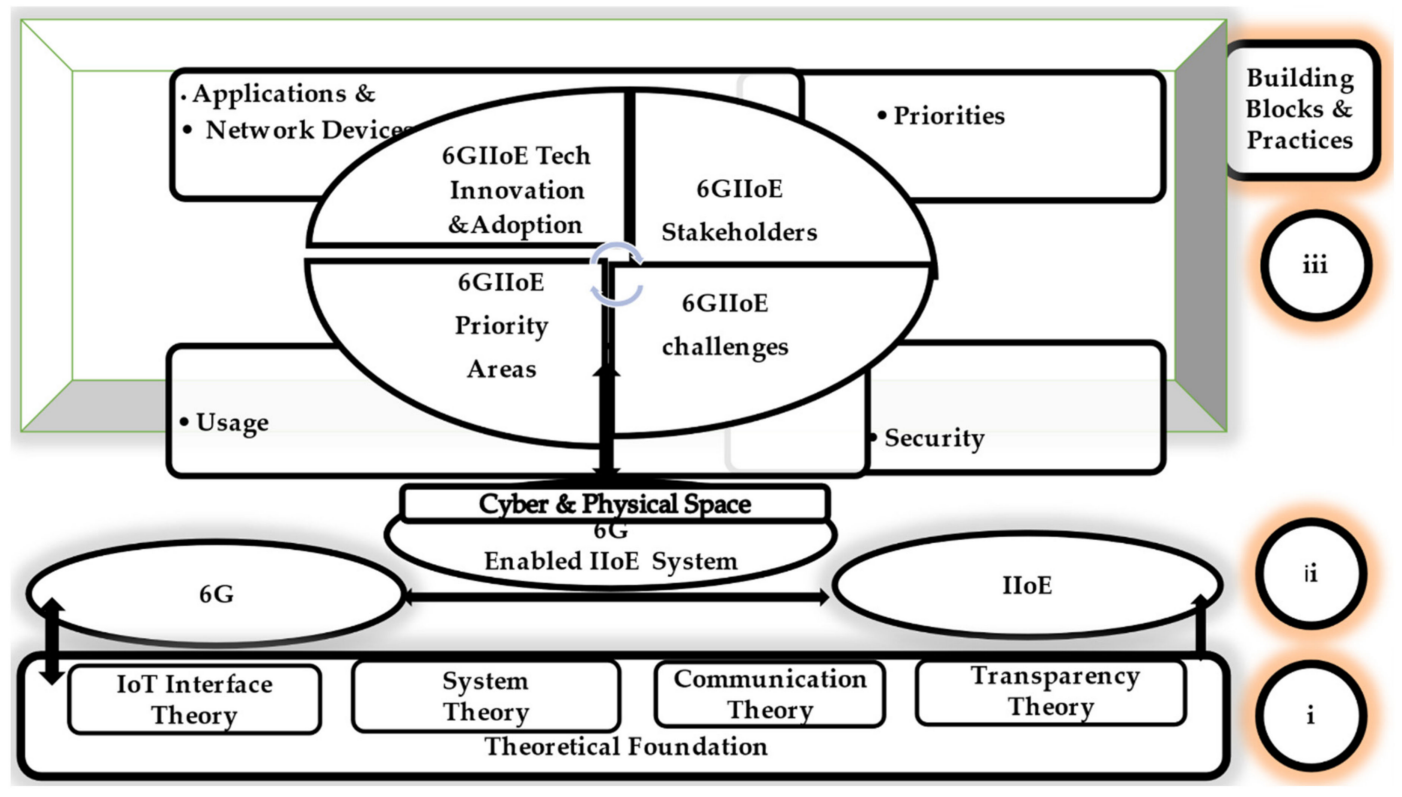

Figure 8. A Novel Theoretical Framework. 
The following are additional contributions of the framework components related to industrial applications, and challenges research:

- $\quad 6$ GIIoE system involves people to machines (P2M), machines communicating with machines (M2M) and people to people (P2P). All stakeholders are paramount for the ultimate success of the 6GIIoE system. Keeping this in mind, cogitation of challenges and priority areas governing to $6 \mathrm{GIIoE}$ system adoption are cavillous and must be addressed cautiously prior to the 6GIIoE execution. This theoretical framework contributes to 6G, and IoE/IIoE knowledge guiding practitioners specifically imparting attention to the challenges of security and privacy to attain trust and successful use of the 6GIIoE system in industrial applications. In view of this contention, we accentuate that as the number of connected devices, sensors, machines increase, at a decisive level, industry professionals need to evaluate the opportunities and threats which the emergence of $6 \mathrm{G}$ enabled IIoE systems might affect to their enterprises indicating that the business models may need to be redefined.

- The theoretical framework outlined in this study aids this aim. The theoretical framework bequeaths to the body of knowledge in the discipline of $6 \mathrm{G}$, IoE, IIoE, and CIIW offering researchers additional explanation and prediction on the theory constituents, thus, crevices opportunities for diversified research. An ample scope for testing this theory and constituents of the theory should be alternatively investigated as well.

\section{Consolidated Lessons Learned}

The following sub-sections present a consolidated lesson learned from this study.

\subsection{Theory Development for IS Innovation}

The study of IS innovation is a complex, multi-dimensional phenomenon with dynamic interactive characteristics that invites a novel theoretical framework. Our primary investigation is to identify a theoretical framework that can accommodate the complex and multidimensional landscape of IS innovation. The prevailing theories related to IS discipline do not adequately account for the dynamic relationship between the innovator and environment. An ecological systems theory can provide an appropriate framework for researchers to approach the topic of innovation and to examine its relationship with information systems. The concepts derived from the literature review can be used to map the layers based on ecological systems theory, which is not within the scope of this study, therefore not covered. It is vitally important to note that IS studies must place the internal organizational processes within the wider philosophical, socioeconomic, and ethical context.

\subsection{G Future Benefits}

We opine that a new set of service classes and IIoE applications will emerge due to $6 \mathrm{G}$ mobile systems. Several cutting-edge technologies will mature along the same time of $6 \mathrm{G}$ deployment to potentially play an important role towards the end of the 6G global standardization. One such prominent technology is quantum computing and communications (QCC) which can provide highly secured long-distance networking in Tera bit per second speed with massive network capacity and extremely low latency. Other 6G technologies will include integration of non-RF and RF links. Industries are motivated to make investments to attain substantial value proposition [53] (p. 1) from 6GIIoE deployment enabling new business models. In addition to the benefits mentioned above, the value drivers of $6 \mathrm{G}$ connectivity for the industries offer the following unique value proposition: (i) high reliability, (ii) greater bandwidth, and (v) operational efficiency. The DNA of 6G value proposition is as follows:

- Human centric services with improved decision making.

- $\quad$ Beyond $6 \mathrm{GHz}$ with tiny high-density cell stations for data bountiful environments.

- Communication with large intelligent surfaces.

- MTRLLC with automation agility. 
- Intelligence efficiency.

- mMTRLLC connectivity latency and reliance

\subsection{IIoE Value Proposition}

IoE are not conventional computing devices. They are physical devices connected to "5G \& Beyond" wireless communication or Wi-Fi or Bluetooth. To operate IoE devices, digital services are required to ensure its systems resiliency. IIoE connectivity unlocks the massive data, enabling enterprises to create new revenue streams, bestow operational efficiencies, cost savings, and enhance customer engagement. To exploit the power of IIoE data one must first tackle connectivity. There are many ways to select the connectivity options and it is daunting to make the right connectivity choices. Multi-National Operators (MNOs) offer different geographical reach; hence collaboration is essential to achieve ubiquitous coverage. A flexible, simple, and cost-efficient service is required to manage the connectivity complexity for enterprises. As the traction of IoE deployments increases exponentially, efficient IoE subscriber management becomes imperative to deploy IIoE projects. Value proposition design, proven IIoE new business models [53] (pp. 1-4) and a simple (user-friendly plug-play) framework are the triumphant combination to create a successful IIoE strategy. The promise of IIoE is endless and the most valuable ones are enhanced customer experience, increased sales revenue, exceptional customer support, recurring revenue stream, and outstanding quality.

In this study, we suggest two tools, as lessons learned, to discover IoE value for industrial applications: (i) the IoE evolution canvas and (ii) the IoE value guide. The following three steps can be executed for industrial applications: (i) collect the data, (ii) make analysis and decision, and (iii) act. The execution can be done in three ways: (i) manually, (ii) assisted, and (iii) automation - the benefit of IoE can be achieved through automation.

IoE value guide is recommended to attain distill opportunities and insights. First, identify and describe IoE value proposition by reviewing the data collection, the analysis and decision making, the appropriate actions (human and machine), and results (customer competitive advantages). Customer benefit primarily depends on data collection, analysis and gathering the insights. The information obtained will create the canvas model and the algorithm required to act. It is vitally important to understand the customer need and determination of return on investment (ROI) in any IIoE project. It is paramount to understand how edge input/output $(\mathrm{I} / \mathrm{O})$ generates a simpler way to meet the needs of IIoE applications. IIoE projects demand special skills and involve many phases to execute them. I/O for the $6 \mathrm{GIIoE}$ projects will pose many challenges and are expensive to execute. To achieve simplicity, security, and cost-effectiveness for your 6GIIoE projects, this study proposes three solutions for improving I/O connectivity: (i) real time (on-time) measurement of equipment and (ii) controlling sensors and devices. It is important to apply the following edge I/O the ideal problem-solver: (i) Web-based configuration, (ii) embedded software, (iii) built-in security.

\subsection{GIIoE System Applications for the CIIW Spectrum}

Each industrial vertical sector of the CIIW will require a different set of 6GIIoE system applications to support its growth with their own unique set of demands. The 6GIIoE technology enablers briefed earlier focused to improve the efficiency of data transmission with low latency and massive network capacity. The exploitation of tiny high-density cell stations, network coding and beamforming will bring key advantages to the data plane. The benefits of a 6GIIoE ecosystem are yet to be well defined for network carriers, smartphone companies, software developers and edge (cloud) infrastructure providers.

\section{Future Research Agenda \& Research Gaps}

Some of the future research areas and/or research gaps regarding IS discipline related to developing theories and 6GIIoE system are grouped into the following categories. 


\subsection{G Enabled IIoE System Theory Development}

A void exists in the literature establishing the theory development on 6G-enabled IoE/IIoE system adoption and execution considering the priority areas, challenges, applications, and enormous opportunities on the horizon for connected intelligent industrial applications. Hence, we suggest researchers enhance knowledge for further studies in the technology areas pertaining to $6 \mathrm{G}$, IoE/IIoE, CIIW to broaden and cultivate a thorough review and theoretical framework constituents where research void or gap prevail.

\subsection{IS Innovations}

IS discipline is well positioned at the intersection of technical, business, and social applications of information communication technology (ICT), operational technology (OT) and its essential dimensions of IIoE. 6GIIoE is emerging as a digital innovation to unleash opportunities in personalized customer experience. The emergence of 6GIIoE heralds a new dimension of a digital destiny with influences that are not yet fully known. This scenario invites the opening of valuable opportunities for scholarly inquiries. Hence, we propose that relevant future research work is essential to develop theories on $6 \mathrm{G}$ and IIoE supporting philosophical, socioeconomic, and ethical dimension specifically for connected intelligent industrial applications to create authentic value for the society at large. Thus, we highlight future research agenda in the following manner:

- Multi-level exploration of IS and heterogeneity.

- Thematic influence domains: (i) impact on organizations; (ii) impact on technology; (iii) impact on individuals; and (iv) impact on society.

\subsection{Smart Service Contracts (SSC)}

While applications are examined related to 6GIIoE ecosystem, there is much more significant potential for using smart service contracts (SSC), distributed ledgers, and other blockchain-related technologies. The research gaps in this domain are the following: (i) increasing the transaction rate per second; (ii) providing architectural means for IoE devices or sensors to utilize the relevant technologies, (iii) allowing secured ways to correct unintended errors in SSC; and (iv) clarification of hidden costs when using blockchains to allow objective comparisons with traditional alternatives [54] (p. 7).

\section{Recommendations}

The following recommendations are based on extensive literature review related to IS, 6G, IoE/IIoE, and CIIW:

\subsection{Theory Development on $6 \mathrm{G}$ enabled IIoE system}

Research scholars place a significant value on theoretical frameworks. Even with such importance, the development of novel theories and depuration of existing theories have been comparatively slighted in the areas of information system (IS) regimen. Validating the scant numbers of research publications on the IS discipline theory development, an exhaustive review of literature presented no research papers found in the literature related to 6GIIoE system. Theories bestow the perception of real-world phenomena and can be perceived as specific ontologies; such as a theory is the nature of real work. Since a void exists in the literature with regard to a theoretical framework for 6GIIoE adoption and execution considering the priority areas, challenges, and applications, we recommend the development of theories related to 6G-enabled IIoE system to enhance research scholars' knowledge for further studies.

\subsection{G Network Specification Requirements}

We suggest the following recommendations for $6 \mathrm{G}$ specification requirements for the connectivity (deep, intelligent, ubiquitous, holographic) vision as outlined below in Section 5 of this study:

- $6 \mathrm{G}$ network application ought to be human centric. 
- High security, and privacy should be key features of 6G.

- Application Types: (i) MTRLLC, (ii) mURLLC, (iii) HCS, and (iv) MPS

- Device Types: (i) Sensors and Devices related CIIW, (ii) Connected Robotics and Autonomous Systems (CRAS), (iii) XR and CBI Equipment, and (iv) Intelligent Plants.

- Architecture: (i) Cell free smart surfaces at High Frequency supported by $\leq 100 \mathrm{~m}$ tiny and dense mmWave cell access, (ii) Hotspots served by Drone carried Base Stations, (iii) Sub-6GHZ cells, and (iv) 5-Layer Platform.

- Frequency Bands: (i)Sub-6 GHZ, (ii) mmWave for mobile access, (iii) Exploration of $\mathrm{THz}$ bands (above $140 \mathrm{GHz}$ ), and (iv) Non-RF (e.g., Optical, Visible Light Communication (VLC) etc.).

- End to End Delay: Less than 1 millisecond

- Processing and Radio only Delay: 10 Nano Seconds

- Rate Requirements: One (1) Tera Bit Per Second.

- Spectral and Energy Efficiency Gains: $1000 \times$ in bps $/ \mathrm{Hz} / \mathrm{m}^{3}$ (volumetric)

- End to End Reliability: Seven 9 Seconds

\subsection{IIoE/Industrial Control Systems (ICS)}

In the IoE era, ICSs [4] (p. 1) are no longer a closed network and suffer from various threats and/or attacks. With the emergence of the fifth industrial revolution, IoE is shifting into IIoE. ICS drives the supervision and management of industrial processes performed by critical infrastructures for industries. The increase in attacks and/or threats influencing ICS drawing attention to the use of Intrusion Detection Systems (IDS) [55]. The malware used to attack critical infrastructure has become increasingly sophisticated and has mimicked normal network traffic. Hence, research pursuit to investigate to protect the threats and/or attacks through machine learning and deep learning with novel detection technique for detecting threats and/or attacks is essential.

\subsection{Securing the 6GIIoE Infrastructure}

The fusion of 6G, IoE, and other emerging digital technologies posing immense new opportunities, and ubiquity experiences. There are many exciting trends that are going to shape the future, inspiring a new age of digital destiny. The insatiable, end-user hunger for network capacity and speed such as $6 \mathrm{G}$ is a key differentiator in a digital journey. Network technologies will ultimately deliver the IIoE. The following are the recommendations for the industries to achieve safe and secure connectivity: (i) manage security at every level of the 6GIIoE system, (ii) protect the identity of the objects and users, (iii) execute multifactor authentication, and (iv) protect identities and not gateways.

\subsection{Cyber Physical System (CPS)}

Most industries are moving into an era of autonomous IIoE and its security must be considered a crucial element. To maintain the emerging growth rate in IIoE, future threats or attacks need the utmost attention. Furthermore, interconnectivity and new application scenarios such as Machine to Machine (M2M) need to be considered. This drives the growth of devices and sensors towards IIoE. The cyber security threats via 6G wireless communications are poised to emerge. Hence, there is a growing demand for the design of new communications systems for Industrial applications. Thus, the industrial world with its fusion of technologies requires Cyber Physical Systems [56]. Hence, research pursuit of resource saving, low cost, and effective alternative to conventional cryptographic methods is imperative to empower confidence in IIoE systems.

\subsection{Security of IIoEvia ICMETRIC Technology}

IIoE holds significant promise for enhanced supply chain efficiencies, quality control, communications, and business productivity for most industries. $6 \mathrm{G}$ is a game changer network technology paradigm that is emerging to tackle the enormous potential of IIoE data associated with the industrial applications. However, the use of IIoE generates new 
vulnerabilities and potential threats. To successfully adopt and execute the 6GIIoE system, it is imperative to improve and enhance the security of $6 \mathrm{G}$ commutations in IIoE. For future industrial manufacturing, the security must be incorporated to deal with the threats by designing it into the IIoE ecosystem at an early stage of the product design life cycle to achieve security in a cost-effective manner. Thus, security of the 6GIIoE applications can be executed by design, utilizing the ICMetric technology [57] that uses hardware and software features as well as the specification of devices or sensors in the IIoE setting.

\section{Conclusions}

Despite inadequate prior research encompassing the IS innovation spectrum to develop theoretical framework, this study developed a novel theoretical framework falls under a theory's focal phenomenon that has not been covered by prior theories. Furthermore, the 6GIIoE priority areas, challenges, applications were identified which offers significant benefits for the connected intelligent industrial applications, and a novel theoretical framework was developed based on the findings and insights. This study has some limitations, specifically the lack of an empirical test of the theoretical model proposed and provides further possible investigation.

Author Contributions: Conceptualization, P.K.P.; Investigation, P.K.P.; Methodology, P.K.P.; Supervision, F.C.-S.; Writing - original draft, P.K.P.; Writing—review \& editing, P.K.P. All authors have read and agreed to the published version of the manuscript.

Funding: This research received no external funding.

Data Availability Statement: MDPI Research Data Policies at https:/ / www.mdpi.com/ethics (accessed on 24 December 2020).

Acknowledgments: The authors would like to greatly thank Fundação para a Ciência e Tecnologia (FCT) and C-MAST (Center for Mechanical and Aerospace Science and Technologies), under project UIDB/00151/2020 for their reviewer support.

Conflicts of Interest: The authors declare no conflict of interest.

\section{References}

1. Ashton, K. That 'Internet of Things' Thing. RFID 2009, 22, 97-114.

2. Alsharif, M.H.; Kelechi, A.H.; Albreem, M.A.; Chaudhry, S.A.; Zia, M.S.; Kim, S. Sixth Generation (6G) wireless networks: Vision, research activities, challenges and potential solutions. Symmetry 2020, 12, 676. [CrossRef]

3. CISCO-Internet of Everything (IoE): Top 10 Insights from Cisco's IoE Value at Stake Analysis for the Public Sector. Available online: http:/ / www.cisco.com/web/about/ac79/docs/IoE/IoE-VAS_Public-Sector_Top-10-\$ \backslash\$Insights.pdf; https:/ / www. cisco.com/web/about/ac79/docs/innov/IoE.pdf (accessed on 17 October 2020).

4. Wang, C.; Wang, B.; Liu, H.; Qu, H. Anomaly detection for industrial control system based on autoencoder neural network. Wirel. Commun. Mob. Comput. 2020, 2020, 1-10. [CrossRef]

5. Singh, P.; Nayyar, A.; Kaur, A.; Ghosh, U. Blockchain and fog based architecture for internet of everything in smart cities. Future Internet 2020, 12, 61. [CrossRef]

6. Viswanathan, H.; Mogensen, P.E. Communications in the 6G Era. IEEE Access 2020, 8, 57063-57074. [CrossRef]

7. Padhi, P.K. 5G \& Beyond: Governance, global policy perspectives, and societal benefits. Int. J. Eng. Technol. Res. Manag. 2020, 4, 87-114.

8. Touching an Intelligent World. Huawei Global Industry Vision (GIV) White Paper. 2018, pp. 1-41. Available online: https: //www.huawei.com/minisite/giv/en/index.html (accessed on 7 December 2020).

9. Siemens Digital Industries Software. 2020. Available online: https://www.plm.automation.siemens.com/global/en/webinar/ track-and-trace-farm-to-table-iot/52777; https:/ / www.plm.automation.siemens.com/global/en/industries (accessed on 8 December 2020).

10. Nord, J.H.; Koohang, A.; Paliszkiewicz, J. The Internet of Things: Review and theoretical framework. Expert Syst. Appl. 2019, 133, 97-108. [CrossRef]

11. Lueth, K.L. The 10 Most Popular Internet of Things Applications Right Now. IoT Analytics. 2015. Available online: https: / / iot-analytics.com/10-internet-of-thingsapplications / (accessed on 21 December 2020).

12. Falkenberg, E.D.; Lyytinen, K.; Verrin-Stuart, A.A. Limitations of information systems theory and practice: A case for plural-ism. In Information System Concepts. IFIP Advances in Information and Communication Technology; Springer: Boston, MA, USA, 1995; pp. 14-15. Available online: http:/ / eprints.lse.ac.uk/2579/1/ISwhatsortofscience.pdf (accessed on 11 December 2020). 
13. Aquilani, B.; Piccarozzi, M.; Abbate, T.; Codini, A.P. The role of open innovation and value co-creation in the challenging transition from industry 4.0 to society 5.0: Toward a theoretical framework. Sustainability 2020, 12, 8943. [CrossRef]

14. Costello, G.J.; Donnellan, B.; Curley, M. A theoretical framework to develop a research agenda for information systems innovation. Commun. Assoc. Inf. Syst. 2012, 33, 26. [CrossRef]

15. Giordani, M.; Polese, M.; Mezzavilla, M.; Rangan, S.; Zorzi, M. Toward 6G networks: Use cases and technologies. IEEE Commun. Mag. 2020, 58, 55-61. [CrossRef]

16. Dang, S.; Amin, O.; Shihada, B. From a Human-Centric Perspective: What Might 6G Be? 2020. Available online: https: / / arxiv.org/pdf/1906.00741.pdf (accessed on 12 December 2020).

17. Huang, C.; Zappone, A.; Alexandropoulos, G.C.; Debbah, M.; Yuen, C. Holographic MIMO Surfaces for 6G Wireless NetWorks: Opportunities, Challenges, and Trends. 2020. Available online: https://arxiv.org/pdf/1911.12296.pdf (accessed on 12 December 2020).

18. Gui, G.; Liu, M.; Tang, F.; Kato, N.; Adachi, F. 6G: Opening new horizons for integration of comfort, security, and intelligence. IEEE Wirel. Commun. 2020, 27, 126-132. [CrossRef]

19. Chowdhury, M.Z.; Shahjalal, M.; Ahmed, S.; Jang, Y.M. 6G wireless communication systems: Applications, requirements, technologies, challenges, and research directions. arXiv 2019, arXiv:1909.11315v1.

20. Mahmood, N.H.; Alves, H.; López, O.A.; Shehab, M.; Osorio, D.P.M.; Latva-Aho, M. Six key enablers for machine type communication in 6G. arXiv 2019, arXiv:1903.05406.

21. Janbi, N.; Katib, I.; Albeshri, A.; Mehmood, R. Distributed Artificial Intelligence-as-a-Service (DAIaaS) for smarter IoE and 6G environments. Sensors 2020, 20, 5796. [CrossRef] [PubMed]

22. Sekaran, R.; Patan, R.; Raveendran, A.; Al-Turjman, F.; Ramachandran, M.; Mostarda, L. Survival study on blockchain based 6g-enabled mobile edge computation for IoT automation. IEEE Access 2020, 8, 143453-143463. [CrossRef]

23. Wang, W.; Liu, F.; Zhi, X.; Zhang, T.; Huang, C. An Integrated deep learning algorithm for detecting lung nodules with low-dose $\mathrm{CT}$ and its application in 6G-enabled internet of medical things. IEEE Internet Things J. 2020, 1. [CrossRef]

24. Yang, P.; Xiao, Y.; Xiao, M.; Li, S. 6G wireless communications: Vision and potential techniques. IEEE Netw. 2019, 33, 70-75. [CrossRef]

25. Saad, W.; Bennis, M.; Chen, M. A Vision of 6G wireless systems: Applications, trends, technologies, and open research problems. IEEE Netw. 2019, 34, 134-142. [CrossRef]

26. Clazzer, F.; Munari, A.; Liva, G.; Lazaro, F.; Stefanovic, C.; Popovsk, P. From 5G to 6G: Has the time for modern random access come? arXiv 2019, arXiv:1903.03063.

27. Tariq, F.; Khandaker, M.R.A.; Wong, K.-K.; Imran, M.A.; Bennis, M.; Debbah, M. A speculative study on 6G. IEEE Wirel. Commun. 2020, 27, 118-125. [CrossRef]

28. Zhang, L.; Liang, Y.-C.; Niyato, D. 6G Visions: Mobile ultra-broadband, super internet-of-things, and artificial intelligence. China Commun. 2019, 16, 1-14. [CrossRef]

29. Internet of Everything (IoE). Internet Everything; IoE: London, UK, 2019; Available online: https://ioe.org/Eppicenter (accessed on 15 December 2020).

30. Dai, H.-N.; Zheng, Z.; Zhang, Y. Blockchain for Internet of Things: A Survey. IEEE Internet Things J. 2019, 6, 8076-8094. [CrossRef]

31. EPPI-Reviewer. Available online: https://eppi.ioe.ac.uk/CMS/Default.aspx?alias=eppi.ioe.ac.uk/cms/er4\& (accessed on 5 December 2020).

32. Swanson, E.B. Information systems innovation among organizations. Manag. Sci. 1994, 40, 1069-1092. [CrossRef]

33. Leidner, D.E.; Kayworth, T. Review: A review of culture in information systems research: Toward a theory of information technology culture conflict. MIS Q. 2006, 30, 357. [CrossRef]

34. Weber, R. Evaluating and developing theories in the information systems discipline. J. Assoc. Inf. Syst. 2012, 13, 1-30. [CrossRef]

35. Chaudhuri, A.; Cavoukian, A. The Proactive and Preventive Privacy (3P) framework for IoT privacy by design. EDPACS 2018, 57, 1-16. [CrossRef]

36. Hsu, C.-L.; Lin, J.C.-C. Exploring factors affecting the adoption of internet of things services. J. Comput. Inf. Syst. 2018, 58, 49-57. [CrossRef]

37. Slepian, D.; Wyner, A.S.O. Rice's contributions to Shannon theory. IEEE Trans. Inf. Theory 1988, 34, 1374. [CrossRef]

38. Lohstroh, M.; Lee, E.A. An Interface Theory for the Internet of Things. Min. Data Financ. Appl. 2015, 20-34. [CrossRef]

39. Cacciapuoti, A.S.; Caleffi, M.; Tafuri, F.; Cataliotti, F.S.; Gherardini, S.; Bianchi, G. Quantum internet: Networking challenges in distributed quantum computing. IEEE Netw. 2019, 34, 137-143. [CrossRef]

40. Averill, E.W.; Gottlieb, J. Two Theories of Transparency; Springer: New York, NY, USA, 2019; pp. 1-21. [CrossRef]

41. Todd, S. A 25-year review of sequential methodology in clinical studies. Stat. Med. 2006, 26, 237-252. [CrossRef] [PubMed]

42. Oughton, E.J.; Frias, Z. The cost, coverage and rollout implications of 5G infrastructure in Britain. Telecommun. Policy 2018, 42, 636-652. [CrossRef]

43. Khan, L.U.; Yaqoob, I.; Imran, M.; Han, Z.; Hong, C.S. 6G wireless systems: A vision, architectural elements, and future directions. IEEE Access 2020, 8, 147029-147044. [CrossRef]

44. Akyildiz, I.F.; Kak, A.; Nie, S. 6G and beyond: The future of wireless communications systems. IEEE Access 2020, 8, 133995-134030. [CrossRef] 
45. Statista Research Department, Internet of Things-Number of Connected Devices Worldwide 2015-2025. Available online: https://www.statista.com/statistics/471264/iot-number-of-connected-devices-worldwide/ (accessed on 17 December 2020).

46. AVANET/ABACUS: Predictive Maintenance with IoT: The Road to Real Returns. Available online: https://www.avnet.com/ wps/portal/abacus/solutions/markets/industrial/predictive-maintenance-iot/ (accessed on 12 December 2020).

47. Kalsoom, T.; Ramzan, N.; Ahmed, S.; Ur-Rehman, M. Advances in sensor technologies in the era of smart factory and industry 4.0. Sensors 2020, 20, 6783. [CrossRef] [PubMed]

48. Riaz, M.N. Implementation of Kanban techniques in software development process: An empirical study based on benefits and challenges. Sukkur IBA J. Comput. Math. Sci. 2019, 3, 25-36. [CrossRef]

49. Roth, N.; Deuse, J.; Biedermann, H. A framework for System Excellence assessment of production systems, based on lean thinking, business excellence, and factory physics. Int. J. Prod. Res. 2019, 58, 1074-1091. [CrossRef]

50. Gochhait, S.; Butt, S.A.; Jamal, T.; Ali, A. Cloud Enhances Agile Software Development; IGI Global: Hershey, PA, USA, 2020; pp. 28-49.

51. Khalid, A.; Butt, S.A.; Jamal, T.; Gochhait, S. Agile scrum issues at large-scale distributed projects. Int. J. Softw. Innov. 2020, 8, 85-94. [CrossRef]

52. Rivard, S. The ions of theory construction. MIS Q. 2014, 38, 3-13.

53. Hudson, D. Value propositions for the internet of things: Guidance for entrepreneurs selling to enterprises. Technol. Innov. Manag. Rev. 2017, 7, 5-11. [CrossRef]

54. Hang, L.; Kim, D.-H. Reliable task management based on a smart contract for runtime verification of sensing and actuating tasks in IoT environments. Sensors 2020, 20, 1207. [CrossRef] [PubMed]

55. Kim, S.; Jo, W.; Shon, T. APAD: Autoencoder-based Payload Anomaly Detection for industrial IoE. Appl. Soft Comput. 2020, 88, 106017. [CrossRef]

56. Chen, B.; Yang, Z.; Huang, S.; Du, X.; Cui, Z.; Bhimani, J.; Xie, X.; Mi, N. Cyber-physical system enabled nearby traffic flow modelling for autonomous vehicles. In Proceedings of the 2017 IEEE 36th International Performance Computing and Communications Conference (IPCCC), San Diego, CA, USA, 10-12 December 2017; pp. 1-6.

57. Tahir, R.; McDonald-Maier, K. ICMetrics based Industrial Internet of Things (IIoT) Security in the Post Quantum World. In Proceedings of the World Congress on Engineering (WCE 2018), London, UK, 4-6 July 2018; Available online: http: / / www.iaeng. org/publication/WCE2018/WCE2018_pp347-348.pdf (accessed on 18 December 2020). 